\title{
August 2012
}

\section{CEPHALOSPORIN-RESISTANT}

NEISSERIA GONORRHOEAE

PUBLIC HEALTH RESPONSE PLAN 


\section{Acknowledgments}

We wish to thank members of the Centers for Disease Control and Prevention (CDC) working group for cephalosporin-resistant gonorrhea response plan at the Division of STD Prevention, National Center for HIV, STD, and TB Prevention, CDC : Ron Ballard, PhD; Stuart Berman, MD, ScM; Russell Cantrell, MPA; Charlotte Kent, PhD; Robert Kirkcaldy, MD, MPH; Kevin O'Connor, MA; John Papp, PhD; Steven J. Shapiro, BS; David Trees, PhD; Hillard Weinstock, MD; Kimberly A. Workowski, MD; Tun Ye, MBBS, PhD; and all external consultants who participated in the consultation meeting on cephalosporin-resistant gonorrhea outbreak response plan, September 14-15, 2009: Mark Aubin, Department of Health, Seattle, WA; Susan Blank, MD, MPH, New York City Department of Health and Mental Hygiene, New York, NY; Gail Bolan, MD, California Department of Public Health, Richmond, CA; Ted Cieslak, MD, DoD Liaison Officer, CDC, Atlanta, GA; Peter Kerndt, MD, Los Angeles County Department of Public Health, Los Angeles, CA; Venie Lee, MS, Hawaii Department of Health, Honolulu, HI; Marc Lipstich, PhD, Harvard School of Public Health, Boston, MA; William G Meyer, Global Emerging Infections Surveillance \& Response (GEIS) Core Dept, DoD, Silver Spring, MD; William L.H. Whittington, University of Washington School of Medicine, Seattle, WA; Dean E. Willis, DrPH, MPH, MA, Florida Department of Health, Jacksonville, FL; William Wong, MD, Division of STI/HIV/AIDS, Chicago, IL.

\section{This document was prepared by Sarah Kidd, Robert Kirkcaldy, Tun Ye, John Papp, David Trees, and Steven J. Shapiro \\ Division of STD Prevention \\ National Center for HIV/AIDS, Viral Hepatitis, STD, and TB Prevention \\ Centers for Disease Control and Prevention}




\section{Guide to Acronyms}

AST

CDC

Ceph-R NG

CLSI

EPT

GISP

MIC

MSM

NAAT

PPNG

QRNG

STD

STI
Antimicrobial Susceptibility Testing

Centers for Disease Control and Prevention

Cephalosporin-Resistant Neisseria gonorrhoeae

Clinical and Laboratory Standards Institute

Expedited Partner Therapy

Gonococcal Isolate Surveillance Project

Minimum Inhibitory Concentration

Men who have Sex with Men

Nucleic Acid Amplification Test

Penicillinase-Producing Neisseria gonorrhoeae

Fluoroquinolone-Resistant Neisseria gonorrhoeae

Sexually Transmitted Disease

Sexually Transmitted Infection 


\section{Contents}

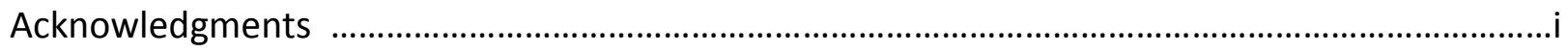

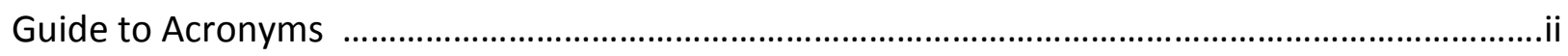

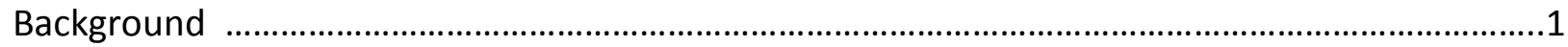

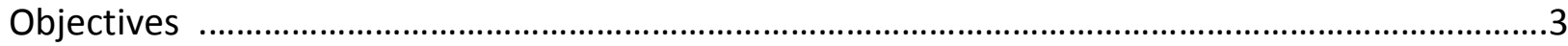

Surveillance for Cephalosporin-Resistant N. gonorrhoeae (Ceph-R NG) …..................................3

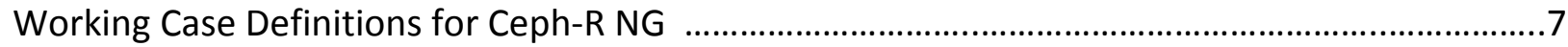

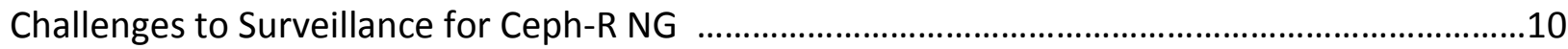

Expansion of Culture and Antimicrobial Susceptibility Testing (AST) Capacity ..........................10

Recommended Clinical Management and Public Health Response Following Detection of

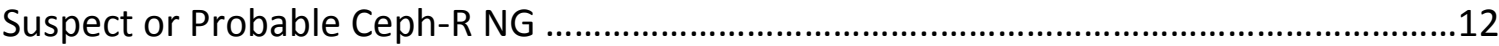

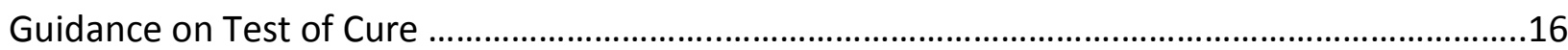

Guidance on Partner Services and Management of Partners of Suspected or Probable

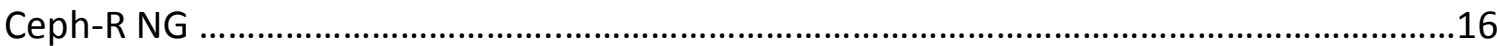

Monitoring the Effectiveness of the Ceph-R NG Response Plan ..................................................17

Role of Enhanced Gonorrhea Prevention and Control Activities .................................................18

Need for Alternative Treatment Options .........................................................................................18

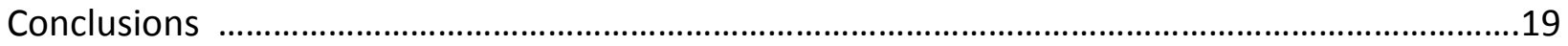

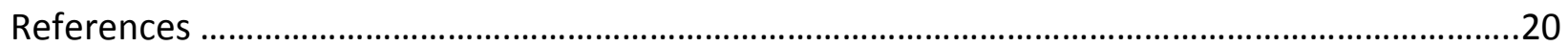

Appendices

I. Contact Information and Selected Web Resources ...................................................22

II. Sample Regulatory Language for Mandated Reporting of AST Results ......................23

III. CLSI cefixime and ceftriaxone agar dilution MIC and disk diffusion zone diameter

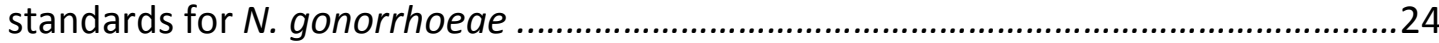

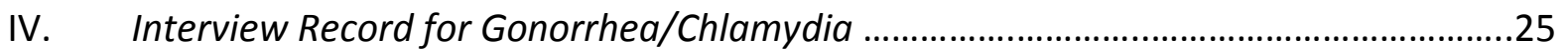




\section{Background}

Gonorrhea is the second most commonly reported notifiable disease in the United States, with 309,341 cases reported in 2010 [1]. Although the national gonorrhea rate decreased $21.7 \%$ during 2000-2010, from 128.7 to 100.8 cases/100,000 population, future progress in gonorrhea control and prevention is threatened by resistance to an increasing number of antimicrobial agents and limited remaining treatment options.

Over the years, Neisseria gonorrhoeae has readily acquired resistance to a broad spectrum of antimicrobial agents traditionally used for the treatment of gonococcal infections. In the United States, the antimicrobial susceptibility patterns of N. gonorrhoeae have been closely monitored since 1986 through the Gonococcal Isolate Surveillance Project (GISP), and the information has been used to update treatment recommendations. In 2010, $27.2 \%$ of all GISP isolates were resistant to penicillin, tetracycline, ciprofloxacin, or some combination of those antimicrobials and $6.9 \%$ of isolates were resistant to all three antimicrobials [1]. Penicillin, tetracycline, and fluoroquinolone antimicrobials are no longer recommended as appropriate treatment for gonorrhea in the United States. Currently, the Centers for Disease Control and Prevention (CDC) recommends dual therapy with ceftriaxone (an injectable cephalosporin) 250 mg intramuscularly as a single dose plus either azithromycin 1 gram orally as a single dose or doxycycline $100 \mathrm{mg}$ orally twice a day for 7 days as the most effective treatment for uncomplicated gonorrhea [2,3]. For patients allergic to cephalosporins, azithromycin 2 grams orally as a single dose is the only alternative treatment option available; however, the use of monotherapy with azithromycin should be extremely judicious due to concerns about possible rapid emergence of azithromycin resistance. Other potential treatment options for gonorrhea are either not available in the United States, do not meet clinical effectiveness criteria for recommended treatment, or have not been adequately studied. Therefore, the emergence and spread of cephalosporin-resistant $N$. gonorrhoeae (Ceph-R NG) would severely limit treatment options for gonorrhea in the United States.

The emergence of Ceph-R NG in the United States could occur either through the importation of Ceph-R NG from other countries, through genetic transformations by obtaining genetic material from other organisms, or through domestic drug selection pressures in the United States. While mutations through drug selection pressures generally take time, the more rapid introduction of antimicrobial-resistant $N$. gonorrhoeae strains to the United States through the importation of resistant strains, followed by subsequent transmission of resistant strains through local sexual networks has always been a concern. 
In the last decade, N. gonorrhoeae strains with decreased susceptibility to cephalosporins have been reported with increasing frequency from countries in Asia and the Pacific region [4-10], as well as from Europe, Canada, and the United States [2, 11, 12]. Cefixime treatment failures were first reported from Japan in 2003 [6] and have subsequently been reported from Norway, the United Kingdom, Austria, and France [13-17]. More recently, and of great concern, a gonococcal isolate with high-level ceftriaxone resistance was identified in a Japanese woman with pharyngeal infection and apparent ceftriaxone treatment failure in 2009 [10]. Gonococcal isolates with high-level ceftriaxone resistance have subsequently been identified in men with urogenital infections in France and in Spain $[17,18]$.

While confirmed cephalosporin treatment failures have not yet been identified in the United States, GISP has detected recent increases in minimum inhibitory concentrations (MICs) for cephalosporins among gonococcal isolates in the United States. From 2006 to the first six months of 2011, the proportion of GISP isolates with an elevated cefixime MIC (MIC $\geq 0.25$ $\mu \mathrm{g} / \mathrm{ml}$ ) increased from $0.1 \%$ to $1.7 \%$, and the proportion with an elevated ceftriaxone MIC (MIC $\geq 0.125 \mu \mathrm{g} / \mathrm{ml}$ ) increased from $0.05 \%$ to $0.5 \%$ [19]. These increases were most notable in the western United States and among MSM. In the West, the proportion of isolates with an elevated cefixime MIC increased from $0.2 \%$ to $3.6 \%$ and the proportion with an elevated ceftriaxone MIC increased from $0.04 \%$ to $1.9 \%$. Among MSM, the proportion with an elevated cefixime MIC increased from $0.2 \%$ to $4.7 \%$, and the proportion with an elevated ceftriaxone MIC increased from $0 \%$ to $1.0 \%$. There is concern that this pattern is reminiscent of the pattern that was seen with the emergence of fluoroquinolone-resistant $N$. gonorrhoeae (QRNG): detection first in Hawaii and then the western United States, before spreading widely in the continental United States. While the first QRNG cases were detected among heterosexuals, QRNG became widespread in the continental United States among men who have sex with men (MSM) with gonorrhea before becoming widespread among heterosexuals [20-22].

It is likely Ceph-R NG infections will emerge in the United States. A significant challenge to developing an effective response strategy is the lack of a successful model for prevention and control of antimicrobial-resistant $N$. gonorrhoeae. In previous efforts to address the emergence of penicillinase-producing N. gonorrhoeae (PPNG) [23] and QRNG [20-22], CDC and the United States public health community have been unable to prevent or control the introduction and spread of antimicrobial-resistant $N$. gonorrhoeae, even when more effective alternative antimicrobial options were available and greater resources were available for patient and partner follow-up.

Limiting the impact of antimicrobial-resistant $N$. gonorrhoeae will require a concerted and sustained effort involving multidisciplinary groups. Although new drug classes or synergistic 
combinations of different antimicrobials may be identified for the treatment of Ceph-R NG and multidrug-resistant $N$. gonorrhoeae, it is critical to prepare for the emergence of Ceph-R NG in the current reality of limited treatment options and limited public health resources. This public health response plan identifies strategies for enhanced surveillance to detect the emergence of Ceph-R NG in the United States and outlines programmatic steps that can be taken to mitigate the impact until cost-effective treatment alternatives can be identified. The effectiveness of this plan should be closely monitored, and the plan should be periodically reviewed and revised, based on updated surveillance, epidemiological and clinical data.

\section{Objectives}

\section{General Objective}

This document is intended to be a resource for state and local health departments in order to minimize the impact of Ceph-R NG on the prevention and control of gonorrhea and its sequelae at the community level in the United States.

\section{Specific Objectives}

(1) To establish enhanced surveillance for patients with suspect or probable Ceph-R NG infections (see case definitions, pages 8-9)

(2) To outline the recommended public health actions to be implemented at the national, state, and local levels following detection of suspect or probable Ceph-R NG cases

\section{Surveillance for Ceph-R NG}

CDC currently conducts surveillance for antimicrobial resistance among $N$. gonorrhoeae isolates through GISP [24]. Since its inception in 1986, GISP has successfully detected trends in resistance, provided prevalence data, and directly informed CDC treatment recommendations for gonorrhea. However, because GISP is a sentinel surveillance system that only conducts surveillance on isolates from symptomatic men attending selected sexually transmitted disease (STD) clinics, and because it takes time to process isolates and measure MICs through GISP's network of reference laboratories, there is a need for additional, more sensitive and timely surveillance approaches in order to detect the emergence of cephalosporin resistance.

The expanded surveillance approach will utilize clinical detection and reporting of suspect and probable Ceph-R NG cases in addition to continued surveillance through GISP. Local 
surveillance could be expanded further by establishing a network of clinics where gonorrhea is commonly diagnosed, and implementing culture-based testing and monitoring of antimicrobial susceptibility of isolates. State and local health departments could also enhance surveillance by monitoring and investigating patients with multiple reports of $N$. gonorrhoeae infection within the preceding 30 days or 60 days to determine whether such reports are possible treatment failures (see possible options for enhancing surveillance, page 5). Together with data from GISP, findings from expanded surveillance will inform future CDC recommendations for gonorrhea treatment, screening, and test of cure.

\section{Clinicians}

Clinicians are requested to (1) maintain vigilance for cephalosporin treatment failures, (2) obtain specimens for culture and antimicrobial susceptibility testing (AST) from patients experiencing possible cephalosporin treatment failure as described below, and (3) report cases that meet the criteria for suspect or probable Ceph-R NG infection (see case definitions, pages 8-9) to the state or local health department.

(1) Detecting possible cephalosporin treatment failure. Cephalosporin treatment failure is the persistence of $N$. gonorrhoeae infection despite appropriate cephalosporin treatment, and could indicate infection with Ceph-R NG. Patients diagnosed with gonorrhea should be educated about Ceph-R NG and instructed to return to care if symptoms do not resolve within 3-5 days or if test of cure is recommended. Patients also should be informed of the need for their partners to be treated and advised to avoid sex for one week following treatment.

Treatment failure due to Ceph-R NG infection should be considered in:

- Patients whose symptoms do not resolve within 3-5 days after appropriate treatment and who report no sexual contact during the post-treatment followup period

- Patients with a positive test of cure (positive culture $\geq 72$ hours or positive nucleic acid amplification test [NAAT] $\geq 7$ days after appropriate treatment) when no sexual contact is reported during the post-treatment follow-up period

- Patients with a positive N. gonorrhoeae culture within 30-60 days (but $\geq 72$ hours) after treatment for gonorrhea who are found to have elevated cephalosporin MICs on AST (see laboratory criteria for suspect or probable CephR NG cases, pages 7-8), regardless of whether sexual contact is reported during the post-treatment follow-up period 
(2) Obtaining specimens for culture and AST. Clinicians should obtain culture specimens for AST from patients with possible treatment failure. In addition, clinicians should also consider obtaining culture specimens from patients who have been diagnosed with another $N$. gonorrhoeae infection in the past 30-60 days, even when re-infection cannot be excluded. Clinicians serving populations at increased risk for gonorrhea (i.e., sexually active patients with one or more of the following risk factors: age $<25$ years, history of previous gonorrhea infection or other sexually transmitted infection, new or multiple sex partners, inconsistent condom use, sex work, or drug use [25]) should establish gonococcal culture procedures in their clinical settings that can be implemented when a case with possible treatment failure is identified.

(3) Reporting. Cases that meet the criteria for suspect or probable Ceph-R NG infection (see case definitions, pages 8-9) should be reported to the state or local health department. Clinicians should also contact the state or local health department if there is concern about Ceph-R NG infection in a patient who does not meet the case definition or if they require assistance obtaining access to culture and AST.

\section{Laboratorians}

Laboratorians are requested to report $N$. gonorrhoeae isolates that meet laboratory-based criteria for suspect or probable Ceph-R NG (see case definitions, pages 8-9) to the state or local health departments.

\section{State and Local Health Departments}

State and local health departments are requested to investigate cases of suspect or probable Ceph-R NG (see case definitions, pages 8-9) and take appropriate public health action, including ensuring that sex partners of case-patients are tested using culture and AST (if culture is positive) and are adequately treated for gonorrhea based on the AST results of the casepatient's isolate. Health departments are requested to report cases of suspect or probable Ceph-R NG to CDC at gispinfo@cdc.gov or (404) 639-8659. State and local health departments are also requested to enhance surveillance for Ceph-R NG cases.

Possible options for enhancing surveillance include:

- Monitoring and investigating patients with multiple laboratory or clinical reports of $N$. gonorrhoeae infections documented within the preceding 30 days or 60 days, to determine whether such reports are duplicative, re-infections, or cephalosporin treatment failures, and following up with possible treatment failures to obtain a test of cure 
- Strengthening of local culture and AST capacity and access (see Expansion of Culture and AST Capacity, page 10)

- Routinely reviewing local N. gonorrhoeae AST results (if available) from the local public health laboratory or commercial laboratories doing AST. Reporting of AST results has been successfully mandated in at least one jurisdiction, and sample regulatory language is available (see Appendix II, page 23).

- Implementing routine test of cure for patients with gonorrhea for prospective case finding of cephalosporin treatment failures

- Establishing a local gonococcal susceptibility surveillance system in a few clinics with a high volume of gonorrhea cases

CDC

CDC will continue to conduct sentinel surveillance of antimicrobial susceptibility through GISP; disseminate surveillance data; provide surveillance, epidemiological, laboratory, clinical and programmatic technical assistance resources and tools to state and local health departments; update treatment recommendations; provide training and technical assistance resources and tools on culture and AST techniques to clinicians and laboratorians; and act as a reference laboratory for confirmatory susceptibility testing. CDC will also work with partners to evaluate and recommend $N$. gonorrhoeae transport media; provide reference strain panels to laboratories for AST quality assurance; evaluate and define the Etest and disk diffusion results that are comparable to the agar dilution MIC breakpoints listed in the Suspect and Probable Ceph-R NG Case Definitions (pages 8-9); evaluate optimal test of cure algorithms; and will continue to collaborate with international partners to enhance and strengthen global surveillance and communication of gonococcal resistance. 


\section{Working Case Definitions for Ceph-R NG}

The working case definitions for Ceph-R NG infection are based on clinical evidence of cephalosporin treatment failure and/or laboratory-based AST results.

Many clinicians do not have access to AST and, thus, the laboratory criteria may not be applicable. However, laboratory criteria are included since laboratory data without clinical outcome data may be available in some instances, such as GISP isolates. Defining laboratory criteria for Ceph-R NG infection is challenging, since the Clinical and Laboratory Standards Institute (CLSI) has not defined MIC breakpoints for resistance [26]. Furthermore, since cephalosporin treatment failures have not been reported yet in the United States, we lack a clear understanding of the relationship between MIC breakpoints and likelihood of treatment failure. The MIC breakpoints for cefixime used in the case definitions below differ from the breakpoints for ceftriaxone because cefixime MICs are generally one-dilution higher than ceftriaxone MICs.

The laboratory criteria in this document refer to cephalosporin MICs obtained by the agar dilution method. CDC is currently in the process of evaluating antimicrobial susceptibility results obtained by the Etest and disk diffusion methods, and is working to establish Etest and disk diffusion breakpoints that are equivalent to the agar dilution breakpoints listed below. Until these data are available, facilities using the Etest method should use the MIC breakpoints listed below (the same breakpoints that are used for agar dilution). Facilities using the disk diffusion method should use the standards published by the CLSI [26] (see table in Appendix III, page 24).

These working case definitions are expected to evolve as new data become available.

\section{Case Description}

A sexually transmitted infection (STI) caused by a cephalosporin-resistant strain of $N$. gonorrhoeae 


\section{Case Classification}

\section{Suspect Case}

A suspect case fulfills either the clinical criteria or laboratory criteria described below:

\section{Suspect Case: Clinical Criteria}

The patient experienced possible cephalosporin treatment failure (symptomatic or asymptomatic) with the following specific components:

- Patient had laboratory-confirmed N. gonorrhoeae infection, and

- Patient received CDC-recommended cephalosporin-based antimicrobial regimen as treatment, and

- Patient subsequently had a positive $N$. gonorrhoeae test result (positive culture $\geq 72$ hours after treatment or positive NAAT $\geq 7$ days after treatment), and

- Patient did not engage in sexual activity after treatment

\section{OR}

\section{Suspect Case: Laboratory Criteria}

AST of pre-treatment or post-treatment isolate of $N$. gonorrhoeae demonstrates:

- Cefixime $\mathrm{MIC} \geq 0.25 \mu \mathrm{g} / \mathrm{ml}$, or

- Ceftriaxone MIC $\geq 0.125 \mu \mathrm{g} / \mathrm{ml}$ 


\section{Probable Case}

A probable case fulfills the clinical and laboratory criteria described below or fulfills the elevated laboratory criteria described below:

\section{Probable Case: Clinical Criteria and Laboratory Criteria}

The patient experienced possible cephalosporin treatment failure (symptomatic or asymptomatic) with the following specific components:

- Patient had laboratory-confirmed N. gonorrhoeae infection, and

- Patient received CDC-recommended cephalosporin-based antimicrobial regimen as treatment, and

- Patient subsequently had a positive $N$. gonorrhoeae test result (positive culture $\geq 72$ hours after treatment or positive NAAT $\geq 7$ days after treatment), and

- Patient did not engage in sexual activity after treatment

\section{AND}

AST of pre-treatment or post-treatment isolate of $N$. gonorrhoeae demonstrates:

- Cefixime MIC $\geq 0.25 \mu \mathrm{g} / \mathrm{ml}$, or

- Ceftriaxone MIC $\geq 0.125 \mu \mathrm{g} / \mathrm{ml}$

\section{OR}

\section{Probable Case: Elevated Laboratory Criteria}

AST of pre-treatment or post-treatment isolate of $N$. gonorrhoeae demonstrates:

- Cefixime MIC $\geq 0.5 \mu \mathrm{g} / \mathrm{ml}$, or

- Ceftriaxone MIC $\geq 0.25 \mu \mathrm{g} / \mathrm{ml}$ 


\section{Challenges to Surveillance for Ceph-R NG}

There are operational difficulties for clinicians, laboratorians and health departments to detect suspect and probable Ceph-R NG cases. The main implementation barriers include:

- Existing surveillance systems that are not likely to be sensitive or timely enough to detect initial emergence. For example, as a sentinel surveillance system, GISP only samples approximately $4 \%$ of male urethral gonorrhea morbidity and usually takes $>1$ month to return a confirmed abnormal MIC result to the sentinel clinic site.

- Lack of clinician's awareness regarding emerging Ceph-R NG

- Lack of clinician's training in the appropriate collection of clinical samples suitable for culture and AST, and lack of quality assurance monitoring

- Lack of clinic availability of laboratory supplies and protocols/procedures for collection and transport of culture specimens (i.e. swab, transport medium, culture plates, $\mathrm{CO}_{2}$ tablets/candle jars and incubators)

- Limited availability of laboratory services for gonococcal culture and AST at the clinical laboratories

- Lack of mandatory laboratory reporting of gonococcal AST results to the local, state, and national public health authorities

- Declining national, state and local STI control resources, personnel and funding

- Test of cure is no longer routine standard for care for management of gonorrhea, so that asymptomatic treatment failures are unlikely to be detected

- Limited data on the expected duration of NAAT-positivity after successful treatment

- Inadequate data on the comparability of agar dilution, Etest, and disk diffusion for AST

\section{Expansion of Culture and AST Capacity}

The reduction of culture and AST capability in the many clinical laboratories is a challenge to achieving timely and effective monitoring of Ceph-R NG. NAAT is now the standard of care for $N$. gonorrhoeae diagnosis, and the availability of routine cultures and AST for $N$. gonorrhoeae is increasingly limited, especially for the increasing proportion of patients seen in settings other than an STD clinic. In most clinics, supplies and proper conditions for gonococcal culture specimen collection (direct inoculation onto selective or non-selective media), storage, and transport (an atmosphere supplemented with $\mathrm{CO}_{2}$ and maintained at $35-37^{\circ} \mathrm{C}$ ) are not available. Access to transport media that adequately maintains the viability of $N$. gonorrhoeae until the specimen reaches a laboratory needs to be established for clinicians, especially those 
working in Federally Qualified Health Centers, primary care, HIV care, corrections facilities, and other clinical settings serving adolescents, young adults, and MSM at high risk for gonorrhea.

A strategic approach to expansion of culture and AST capacity is required to support the proposed enhanced surveillance and response activities for Ceph-R NG. The strategy should include:

\section{At state and local health departments:}

- Local assessment by health departments of commercial and public health laboratory capacity in gonococcal culture and AST

- In locales that lack culture and AST capabilities, establishment of partnerships with laboratories that can provide culture and AST. These partnerships may include the establishment of local, state, or regional networks to support culture and AST.

- Local health department facilitation of access to gonococcal culture, especially in clinics serving patients at risk for gonorrhea or from where a high volume of gonorrhea cases are reported, either through developing referral systems with documented linkage to culture for local clinicians, or facilitation of local availability of $N$. gonorrhoeae culture plates, transport media and appropriate transport supplies to clinicians

- Facilitation of local training for clinicians and laboratorians

\section{At CDC:}

- Assist with assessment of state and local health department access to gonococcal culture and AST

- Evaluation of existing transport media (e.g., Amies charcoal, Transgrow, InTray ${ }^{\mathrm{TM}}$ ) and identification or development of transport media that maintain $N$. gonorrhoeae viability and that can be used in the non-STD clinic setting or in other clinic settings where appropriate culture plates, candle jars, and incubators are not available

- Development of training and technical assistance resources (website materials, protocols, and manuals) on specimen collection, transport, culture \& AST techniques and implementation of website-based training activities, in collaboration with public (e.g. Association of Public Health Laboratories) and private sector partners (e.g., Quest, LabCorp, and Kaiser)

At this time, the molecular mechanisms associated with cephalosporin resistance have not been fully elucidated. Laboratory studies to improve our knowledge-base of molecular and biologic mechanisms of resistance should be continued and might contribute to the development of molecular detection methods for resistant organisms. A point-of-care 
molecular test for detection of resistance would dramatically enhance our national response to cephalosporin resistant and multi-drug resistant gonorrhea.

\section{Recommended Clinical Management and Public Health Response Following Detection of Suspect or Probable Ceph-R NG}

\section{Clinician Responsibilities}

Cefixime treatment failures. When a clinician identifies a patient with gonorrhea and possible treatment failure (see Detecting possible cephalosporin treatment failure, page 4) following treatment with an oral cephalosporin (i.e., cefixime), he/she should:

- Based on anatomic exposure history, obtain specimens for culture from pharyngeal, rectal, and genital sites for AST. Clinicians should notify the laboratory that the specimen was obtained from a patient with possible cephalosporin treatment failure.

- Re-treat with $250 \mathrm{mg}$ of ceftriaxone as a single intramuscular dose and azithromycin 2 grams orally as a dual therapy

- Ensure eradication of infection with a test of cure after re-treatment, preferably with culture ( $\geq 72$ hours after re-treatment), or if culture is not available, with NAAT ( $\geq 7$ days after re-treatment). If the test of cure NAAT is positive, a specimen for culture should be obtained to both ensure that the NAAT result is reliable for active infection and to allow for AST.

- Test the patient's sex partners from the two months preceding the patient's initial gonorrhea diagnosis date (preferably with culture from all exposed sites) and empirically treat these partners with ceftriaxone $250 \mathrm{mg}$ intramuscularly and azithromycin 2 grams orally as dual therapy

- Promptly notify the state or local health departments within 24 hours of identification

Ceftriaxone treatment failures. When a clinician identifies a patient with gonorrhea and possible treatment failure (see Detecting possible cephalosporin treatment failure, page 4) following treatment with an injectable cephalosporin (i.e., ceftriaxone), he/she should:

- Consult with a local infectious diseases expert or STD/HIV Prevention Training Center (see www.nnptc.org for regional contact information), and with the state or local health department, regarding re-treatment and partner management. The state or local health departments should inform CDC. 
- Based on anatomic exposure history, obtain specimens for culture from pharyngeal, rectal, and genital sites for AST. Clinicians should notify the laboratory that the specimen was obtained from a patient with possible cephalosporin treatment failure.

- Ensure eradication of infection with a test of cure after re-treatment, preferably with culture ( $\geq 72$ hours after re-treatment), or if culture is not available, with NAAT ( $\geq 7$ days after re-treatment). If the test of cure NAAT is positive, a specimen for culture should be obtained to both ensure that the NAAT result is reliable for active infection and to allow for AST.

- Test the patient's sex partners from the two months preceding the patient's initial gonorrhea diagnosis date (preferably with culture from all exposed sites) and empirically treat these partners with the same antimicrobial regimen with which the index patient was re-treated and which most likely will cure him/her (based on consultation with a local infectious disease expert, state or local health department, and CDC)

- Promptly notify the state or local health department within 24 hours of identification

\section{Laboratorian Responsibilities}

Laboratorians who identify a N. gonorrhoeae isolate with AST results that are consistent with suspect or probable Ceph-R NG (see Case Definitions, pages 8-9) should promptly notify (within 24 hours) the ordering clinician as well as the state or local health department. Results from isolates associated with possible clinical treatment failures should also be promptly reported. Culture specimens obtained from all patients with possible treatment failure or suspect or probable Ceph-R NG infection should be sent to CDC for AST by agar dilution and stored at the local laboratory and/or CDC in case further testing is needed.

Testing and/or storage of specimens or isolates at CDC should be facilitated by the state or local health department according to local public health protocols. Instructions for shipping isolates to $\mathrm{CDC}$ can be found at:

www.cdc.gov/std/Gonorrhea/arg/specimen_shipping_instructions1-29-08.pdf

N. gonorrhoeae is classified as Category B (UN 3373).

\section{State and Local Health Department Responsibilities}

When notified by a clinician or laboratorian of gonorrhea case with possible treatment failure or with suspect or probable Ceph-R NG infection, local public health authorities should initiate an investigation to collect the clinical and epidemiological information outlined in the Interview Record for Gonorrhea/Chlamydia (see Appendix IV, page 25, or http://www.cdc.gov/std/Program/forms/GC_CT_IR_6_05_2008.pdf and http://www.cdc.gov/std/Program/forms/GC-CT/GC_CT-IRInstructions.pdf for instructions) from the index case and sexual partner(s). Locally developed interview instruments that collect 
these data can be used. Data elements that state and local health departments should consider collecting during an investigation are outlined below, and health departments should aim to gather these data within 30 days of the identification of a case. State and local health departments are requested to promptly notify (within 24 hours) CDC's Division of STD Prevention (at (404) 639-8659 or gispinfo@cdc.gov) of suspect or probable Ceph-R NG cases (see case definitions, pages 8-9), or if there is concern about Ceph-NG infection in a patient who does not meet the case definition.

\section{Epidemiological Investigation of Suspect or Probable Ceph-R NG Cases}

All cases and sex partners who can be traced should be interviewed to identify the demographic characteristics, sexual behavior, and risk factors outlined on the Interview Record for Gonorrhea/Chlamydia (see Appendix IV, page 25, or www.cdc.gov/std/Program/forms/GC_CT_IR_6_05_2008.pdf). Ideally, other persons within the patient's socio-sexual network should be interviewed also.

The Interview Record for Gonorrhea/Chlamydia includes the following data elements for cases:

- Age, sex at birth, race, and ethnicity

- Address of residence, contact information

- Provider and facility type where tested

- Date(s) of test(s), type of test(s), source(s) of specimen(s) (anatomic site), and result of test(s)

- Treatment date(s), drug(s) and dosage(s) given

- Gender of sex partners in last 12 months

- History of anonymous sex in the last 12 months

- History of incarceration in the last 12 months

- History of injection or non-injection drug use in the last 12 months

- Method of case detection, reason for seeking care

- HIV status

- History of signs or symptoms

- STD history

- Type of partner services offered and provided

- Place where patient met his/her partner(s)

- Incidental antimicrobial treatment in the last 12 months, treatment dates, drug and dosage, and reason for use 
In addition, it is recommended that state and local health departments collect the following data elements during interviews with a suspect or probable Ceph-R NG case and partner(s) and medical record review:

- Type of sex (oral, vaginal, and/or anal; insertive and/or receptive)

- Travel history during past 3 months (including place and whether the patient had sex during travel)

- Travel history of sex partners in the past 3 months (if known) (including place and whether the partner had sex during travel)

- Results of AST (if available and performed)

- History of commercial sex work (involvement in or solicitation of)

- Incarceration of sex partners

Below are additional epidemiological and descriptive investigations to consider if a Ceph-R NG case is identified and if local capacity exists. CDC can provide technical assistance for such investigations.

\section{Evaluate epidemiological characteristics associated with Ceph-R NG strains:}

- Case-control study comparing demographic and risk behaviors between Ceph-R NG cases and cephalosporin-susceptible cases

- Geospatial analysis of cases

\section{Evaluate epidemiological or network linkages between cases:}

- Interview index cases, sex partners, and sex partners of partners to establish network linkages of people and places/venues/internet sites/smart-phone apps

- Conduct strain typing of isolates to assess microbiological linkages.

\section{Evaluation of estimated local prevalence of Ceph-R NG:}

- Review case-based surveillance data to identify repeat infections in a single person within a short time frame (e.g., within preceding 30 to 60 days)

- Assess local AST capacity and review local AST results

- Systematically collect local specimens for culture and AST

- Implement routine test of cure for prospective local surveillance for Ceph-R NG cases 


\section{Guidance on Test of Cure}

Patients treated for gonorrhea who (1) have symptoms which fail to resolve within 3-5 days after appropriate treatment, (2) are found to have AST results consistent with the laboratory criteria for suspect or probable Ceph-R NG (see case definitions, pages 8-9), (3) are treated with an alternative regimen (i.e., a regimen other than ceftriaxone $250 \mathrm{mg}$ intramuscularly plus either azithromycin $1 \mathrm{gm}$ orally as a single dose or doxycycline $100 \mathrm{mg}$ orally twice a day for 7 days) should receive a test of cure.

In addition, if clinicians or health departments are concerned about local emergence of Ceph-R NG based on available local GISP and epidemiological data, they might consider implementing or recommending routine test of cure 7-10 days after treatment for all gonorrhea cases for a period of time to determine yield. Routine test of cure may allow for rapid identification of treatment failures and provide timely prevalence estimates of cephalosporin resistance.

If done, test of cure is preferably performed with culture ( $\geq 72$ hours after treatment), but may be performed with NAAT ( $\geq 7$ days after treatment), if culture is not available. The possibility of false-positivity with NAAT as early as 7 days after treatment is a concern, but is likely to be low [27]. However, further study on the feasibility and cost-effectiveness of this approach is warranted, and the recommendations on timing of test of cure are expected to evolve as new data become available. At present, asymptomatic patients with a positive NAAT on test of cure should be cultured. While awaiting the culture result, these patients should be managed according to clinical history and reported if they meet the current criteria for a suspect or probable Ceph-R NG case (see case definitions, pages 8-9).

\section{Guidance on Partner Services and Management of Partners of Suspected and Probable Ceph-R NG}

Providers should discuss with their patients who have suspect or probable Ceph-R NG infection the critical need for their sex partners to seek medical evaluation and treatment, and the role of health department assistance regarding linking partners to care and treatment.

Clinicians and health departments should make strenuous efforts to link partners to care. Partners of patients with suspect or probable Ceph-R NG infection should be prioritized for Disease Intervention Specialist partner services by state and local health departments, and 
should be prioritized for urgent clinical care at STD clinics or other settings so they can be tested and treated appropriately.

All sex partners of suspect or probable Ceph-R NG cases from the two months preceding the index patient's initial test date (i.e., date of diagnosis) should be tested for $N$. gonorrhoeae infection (preferably with culture from all exposed anatomic sites), and empirically treated as follows: Sex partners of patients with possible cefixime treatment failure should be treated with 250 mg ceftriaxone intramuscularly and azithromycin 2 grams orally in a single dose. Sex partners of patients with possible ceftriaxone treatment failure should be treated with the same antimicrobial regimen with which the index patient was re-treated and which most likely will cure him/her (based on consultation with a local infectious disease expert or STD/HIV Prevention Training Center, state or local health department, and CDC). All sex partners should be tested and re-treated as described above, even if they were previously treated with oral cefixime.

State and local health departments and CDC should continue to evaluate the role and advisability of expedited partner therapy (EPT) after the emergence of Ceph-R NG. Because local susceptibility data is unlikely to be collected systematically, decision-making may be based upon regional GISP data. Exposed sex partners should be informed that dual therapy with ceftriaxone and either azithromycin or doxycycline is the most effective treatment for gonorrhea, and should be strongly advised to present to a clinic for dual treatment that includes ceftriaxone. However, for heterosexual patients with gonorrhea whose partners are unlikely to seek evaluation and treatment, EPT using cefixime and either azithromycin or doxycycline can be considered. This approach should always be accompanied by efforts to encourage partners to seek clinical evaluation and to educate partners about the need for test of cure if a cefixime-based regimen is used.

\section{Monitoring the Effectiveness of the Ceph-R NG Response Plan}

State and local health departments should monitor the effectiveness of Ceph-R NG response activities by establishing state and local program improvement indicators. Positive improvements could be indicated by:

- Increases in number of laboratories with N. gonorrhoeae culture and AST capacity

- Increases in number of clinics with appropriate transport media and specimen collection and transport procedures 
- Improvements in level of awareness on the emergence of Ceph-R NG and recommended public health response actions among clinicians and laboratorians

- Increases in timely reporting and investigation of suspect or probable Ceph-R NG cases

- Increases in the proportion of gonorrhea patients treated with CDC-recommended regimen: ceftriaxone $250 \mathrm{mg}$ intramuscularly plus either azithromycin $1 \mathrm{gm}$ orally as a single dose or doxycycline $100 \mathrm{mg}$ orally twice a day for 7 days

- Other surveillance system indicators such as sensitivity, specificity, timeliness in detecting suspect or probable Ceph-R NG cases

CDC should assess the overall impact of national Ceph-R NG public health response action by monitoring local access to culture/AST and the trends of emergence and transmission of Ceph-R NG strains in the United States by using epidemiology and mathematical modeling approaches.

\section{Role of Enhanced Gonorrhea Prevention and Control Activities}

While the clinical and public health responses recommended by the Ceph-R NG Response Plan largely focus on targeted intervention activities for suspect or probable Ceph-R NG cases, public health authorities should also continue to scale up general gonorrhea prevention and control activities. These activities are designed to reduce the overall gonorrhea disease burden through prevention, early diagnosis, timely and effective treatment, and partner services. Effective gonorrhea prevention and control activities will reduce overall disease burden and thus the response needed when Ceph-R NG strains are identified.

\section{Need for Alternative Treatment Options}

Currently, CDC recommends dual therapy with ceftriaxone $250 \mathrm{mg}$ intramuscularly as a single dose with either azithromycin 1 gram orally as a single dose or doxycycline $100 \mathrm{mg}$ orally twice a day for 7 days for the treatment of uncomplicated gonorrhea [2, 3]. Based on available data, this is the most effective regimen against urogenital, rectal, and pharyngeal gonorrhea; however, it is not known whether use of this dual regimen can delay the emergence of Ceph- $R$ NG.

In the past, $\mathrm{CDC}$ recommendations for gonorrhea treatment have been based on the criteria of efficacy in $\geq 95 \%$ of $N$. gonorrhoeae infections in the population [28]. When resistance developed to recommended treatment regimens in the past, the public health response was to 
switch to an alternative class of antimicrobials that would effectively treat $\geq 95 \%$ of $N$. gonorrhoeae strains in the United States. The current lack of well-studied and effective therapy for Ceph-R NG is a major challenge for public health authorities in responding to the threat of emerging Ceph-R NG.

Effective alternative antimicrobials or antimicrobial combinations for the treatment of gonorrhea are needed urgently; thus, the development of novel antimicrobials and clinical trials to study combinations of existing antimicrobials is necessary.

In collaboration with international and national partners, CDC will develop an evidence base of the effectiveness of existing antimicrobials and drug combinations and support the development and evaluation of novel antimicrobial agents and drug combinations that are ideally oral, single dose, meet the $95 \%$ effectiveness standard, and are not cost-prohibitive. CDC will work with the National Institutes of Health (NIH) and the new National Center for Advancing Translational Sciences (NCATS) to develop additional gonorrhea treatment options. CDC will also work with professional organizations concerned with antimicrobial resistance, such as Infectious Diseases Society of America (IDSA), the Association of Public Health Laboratories (APHL), and the National Coalition of STD Directors (NCSD). Lastly, CDC will continue to work with WHO, especially in the implementation of the Global Action Plan [29], as Ceph-R NG is a global public health concern.

\section{Conclusions}

This response plan is prepared as a pro-active attempt to minimize the impact of Ceph-R NG through the implementation of effective clinical services and public health prevention practices. As more evidence becomes available, the case definitions, clinical management, and public health response strategies outlined in this document will need to be reviewed and revised. Barriers to the effective control of Ceph-R NG exist, and in an era of declining STI public health infrastructure, local, state, and federal resources may need to be reprioritized to implement this plan. However, a coordinated and sustained effort that includes clinicians, laboratorians, and state and local health departments will limit the impact of antimicrobial-resistant $N$. gonorrhoeae. Preparation and action now, prior to the emergence of resistant strains, will allow for a more effective and less costly response later. 


\section{References}

[1] Centers for Disease Control and Prevention. Sexually Transmitted Disease Surveillance 2010. Atlanta: U.S. Department of Health and Human Services, 2011.

[2] Centers for Disease Control and Prevention. Cephalosporin susceptibility among Neisseria gonorrhoeae isolates - United States, 2000-2010. MMWR 2011;60(26):873-7.

[3] Centers for Disease Control and Prevention. Update to CDC's sexually transmitted diseases treatment guidelines, 2010: Oral cephalosporins no longer recommended for treatment of gonococcal infections. MMWR in press.

[4] Akasaka S, Muratani T, Yamada Y, et al. Emergence of cephem- and aztreonam-high resistant Neisseria gonorrhoeae that does not produce $\beta$-lactamase. J Infect Chemother 2001;7:49-50.

[5] Deguchi T, Yasuda M, Yokoi S, et al. Treatment of uncomplicated gonococcal urethritis by double-dosing of $200 \mathrm{mg}$ cefixime at a 6-h interval. J Infect Chemother 2003;9:35-9.

[6] Yokoi S, Deguchi T, Ozawa T, et al. Threat to cefixime treatment for gonorrhea. Emerg Infect Dis 2007;13(8):1275-7.

[7] Lo JYC, Ho KM, Leung AOC, et al. Cefibuten resistance and treatment failure of Neisseria gonorrhoeae infection. Antimicrob Agents Chemother 2008;52(10):3564-7.

[8] Ito M, Yasuda M, Yokoi S, et al. Remarkable increase in central Japan in 2001-2002 of Neisseria gonorrhoeae isolates with decreased susceptibility to penicillin, tetracycline, oral cephalosporins, and fluoroquinolones. Antimicrob Agents Chemother 2004;48(8):3185-7.

[9] Su X, Jiang F, Quimuge, et al. Surveillance of antimicrobial susceptibilities in Neisseria gonorrhoeae in Nanjing, China, 1999-2006. Sex Transm DIs 2007;34(12):995-9.

[10] Ohnishi M, Saika T, Hoshina S, et al. Ceftriaxone-resistant Neisseria gonorrhoeae, Japan. Emerg Infect Dis 2011;17:148-9.

[11] Cole MJ, Chisholm SA, Hoffmann S, Stary A, Lowndes CM, Ison CA, et al. European surveillance of antimicrobial resistance in Neisseria gonorrhoeae. Sex Transm DIs 2010;86:427-32.

[12] Martin I, Swatzky P, Allen V, Hoang L, Lefebvre B, Mina N, et al. Emergence and characterization of Neisseria gonorrhoeae isolates with decreased susceptibilities to ceftriaxone and cefixime in Canada: 2001-2010. Sex Transm DIs 2012;39(4):316-23.

[13] Unemo M, Golparian D, Syversen G, et al. Two cases of verified clinical failures using internationally recommended first-line cefixime for gonorrhoea treatment, Norway, 2010. Eurosurv 2010;15(47).

[14] Ison CA, Hussey J, Sankar KN, et al. Gonorrhoea treatment failures to cefixime and azithromycin in England, 2010. Eurosurv 2011;16(14).

[15] Forsyth S, Penney P, Rooney G. Cefixime-resistant Neisseria gonorrhoeae in the UK: a time to reflect on practice and recommendations. Internat J STD \& AIDS 2011;22:296-7.

[16] Unemo M, Golparian D, Stary A, Eigentler A. First Neisseria gonorrhoeae strain with resistance to cefixime causing gonorrhoeae treatment failure in Austria, 2011. Euro Surveill 2011;16(43):pii=19998.

[17] Unemo M, Golparian D, Nicholas R, Ohnishi M, Galay A, Sednaoui P. High-level cefixime- and ceftriaxone-resistant N. gonorrhoeae in Europe (France): novel penA mosaic allele in a successful international clone causes treatment failure. Antimicrob Agents Chemother 2012;56(3):1273-80.

[18] Camara J, Serra J, Ayats J, Bastida T, Carnicer-Pont D, Andreu A, et al. Molecular characterization of two high-level ceftriaxone-resistant Neisseria gonorrhoeae isolates detected in Catalonia, Spain. J Antimicrob Chemother 2012;Epub ahead of print.

[19] Bolan GA, Sparling PP, Wassherheit JN. The emerging threat of untreatable gonococcal infection. N Engl J Med 2012;366(6):485-7. 
[20] Centers for Disease Control and Prevention. Increases in fluoroquinolone-resistant Neisseria gonorrhoeae -- Hawaii and California, 2001. MMWR 2002;51:1041-4.

[21] Centers for Disease Control and Prevention. Increases in fluoroquinolone-resistant Neisseria gonorrhoeae among men who have sex with men -- United States, 2003, and revised recommendations for treatment, 2004. MMWR 2004;53:335-8.

[22] Centers for Disease Control and Prevention. Sexually Transmitted Diseases Treatment Guidelines, 2006. MMWR 2006;55(RR-11).

[23] Centers for Disease Control and Prevention. Antibiotic-resistant strains of Neisseria gonorrhoeae. Policy guidelines for detection, management, and control. MMWR 1987;36(5S).

[24] Schwarz S, Zenilman J, Schnell D, et al. National surveillance of antimicrobial resistance in Neisseria gonorrhoeae. JAMA 1990;264:1413-7.

[25] U.S. Preventive Services Task Force. Screening for gonorrhea: recommendation statement. Ann Fam Med 2005;3:263-7.

[26] Clinical and Laboratory Standards Institute. Performance standards for antimicrobial susceptibility testing. Twenty-first informational supplement. CLSI document M100-S21. Wayne, PA: Clinical and Laboratory Standards Institute, 2011.

[27] Bachmann LH, Desmond RA, Stephens J, et al. Duration of persistence of gonococcal DNA detected by ligase chain reaction in men and women following recommended therapy for uncomplicated gonorrhea. J Clin Microbiol 2002;40(10):3596-601.

[28] Handsfield HH, McCutchan JA, Corey L, Ronald AR. Evaluation of new anti-infective drugs for the treatment of uncomplicated gonorrhea in adults and adolescents. Clin Infect Dis 1992;15(Suppl 1):S12330.

[29] World Health Organization. Global action plan to control the spread and impact of antimicrobial resistance in Neisseria gonorrhoeae. Geneva, Switzerland: World Health Organization, 2012. 


\section{Appendix I:}

\section{Contact Information and Selected Web Resources}

To report cases of suspect or probable Ceph-R NG to CDC:

Email gispinfo@cdc.gov or call (404)639-8659

To consult with a STD/HIV Prevention Training Center:

Visit www.nnptc.org for the contact information of the Training Center closest to you

Instructions for shipping isolates to CDC can be found at:

www.cdc.gov/std/Gonorrhea/arg/specimen_shipping_instructions1-29-08.pdf

Electronic copies of the Interview Record for Gonorrhea/Chlamydia and instructions can be found at:

http://www.cdc.gov/std/Program/forms/GC_CT_IR_6_05_2008.pdf

http://www.cdc.gov/std/Program/forms/GC-CT/GC_CT-IRInstructions.pdf

STD Treatment Guidelines:

http://www.cdc.gov/STD/treatment/ 


\section{Appendix II:}

\section{Sample Regulatory Language for Mandated Reporting of Antimicrobial Susceptibility Testing (AST) Results}

The following is an excerpt from New York City's regulations concerning reportable conditions:

"The director of a clinical laboratory conducting an examination of a specimen submitted for analysis shall, except as noted below with respect to blood banks, report to the Department, within 24 hours of obtaining results, all positive or reactive laboratory findings which indicate the presumptive or confirmed presence of any disease or condition required to be reported by subdivision (a) of $\S 11.03$ of this Code, and also any laboratory findings which are otherwise required to be reported pursuant to this Article; ... Reports shall contain all of the information and data elements required by the reporting forms or electronic reporting format approved by the Department, including but not limited to: ... (8) The antibiotic susceptibility testing results for bacterial diseases listed under subdivision (a) of $\S 11.03$ of this Code. This requirement includes traditional broth, agar and newer automated methods of antibiotic susceptibility testing, as well as molecular-based methods that assay for molecular determinants of antibiotic resistance."

Reference: New York City, N.Y., Tit. 24, Health Code, § 13.03. 


\section{Appendix III: \\ Table: CLSI cefixime and ceftriaxone agar dilution MIC and disk diffusion zone diameter standards for $\mathbf{N}$. gonorrhoeae [26]}

\begin{tabular}{lcc}
\hline Antimicrobial agent & MIC by agar dilution & Zone diameter by disk diffusion \\
\hline Cefixime & $\geq 0.25 \mu \mathrm{g} / \mathrm{ml}$ & $\leq 31 \mathrm{~mm}$ \\
& $\geq 0.5 \mu \mathrm{g} / \mathrm{ml}$ & Not defined \\
Ceftriaxone & $\geq 0.125 \mu \mathrm{g} / \mathrm{ml}$ & Not defined \\
& $\geq 0.25 \mu \mathrm{g} / \mathrm{ml}$ & $\leq 35 \mathrm{~mm}$ \\
\hline
\end{tabular}




\section{Appendix IV: \\ Interview Record for Gonorrhea/Chlamydia \\ and \\ Instructions for Interview Record for Gonorrhea/Chlamydia}


Interview Record for Gonorrhea/Chlamydia

Patient ID Condition(s)

Relnfection? If yes, \#

Case ID

Interview
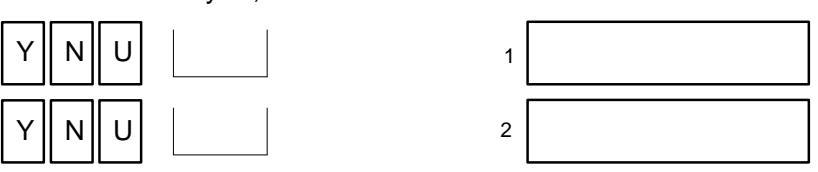

Record ID

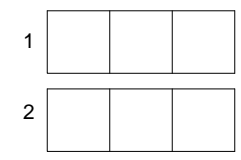

Name

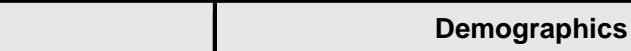

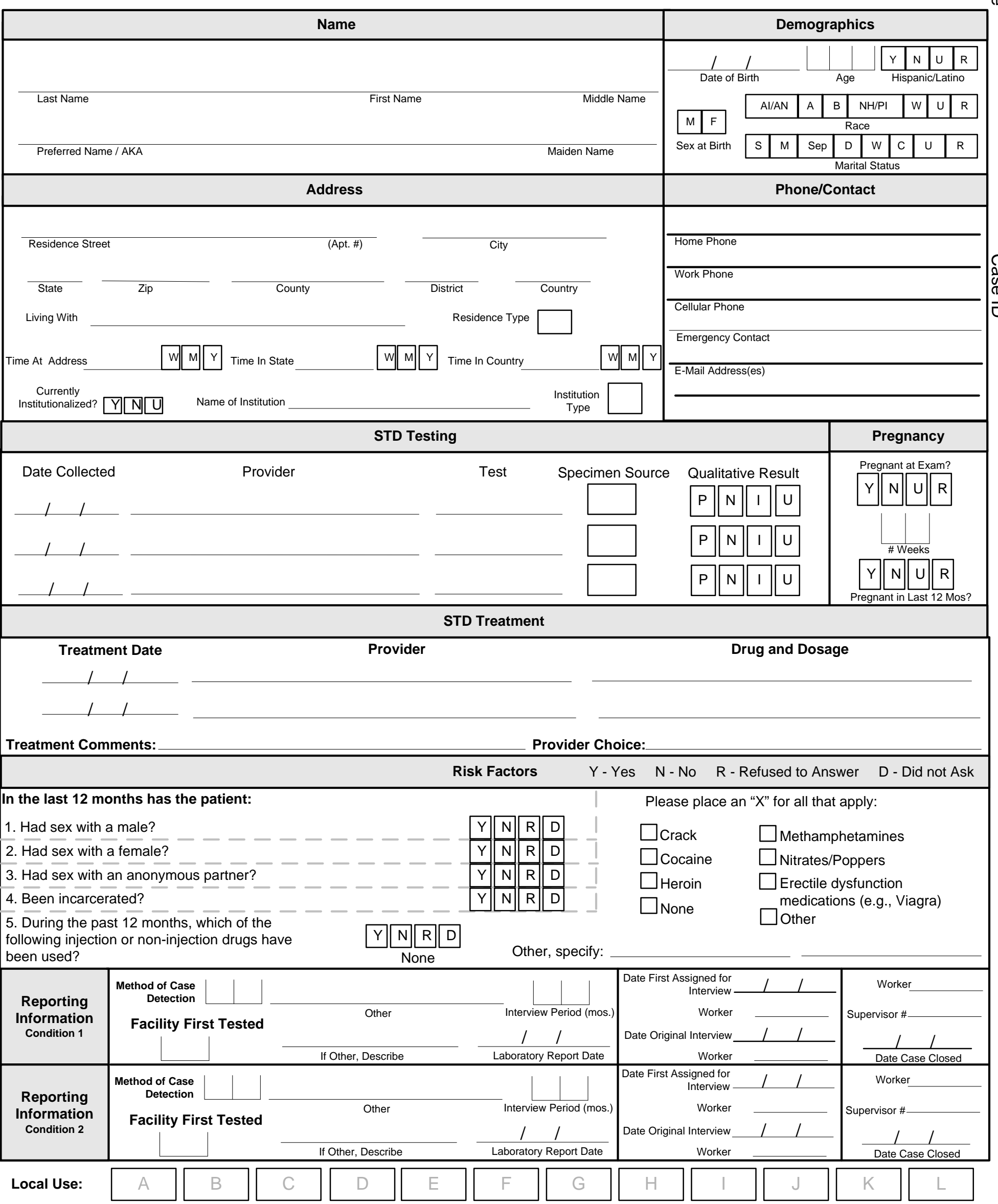


HIV Testing

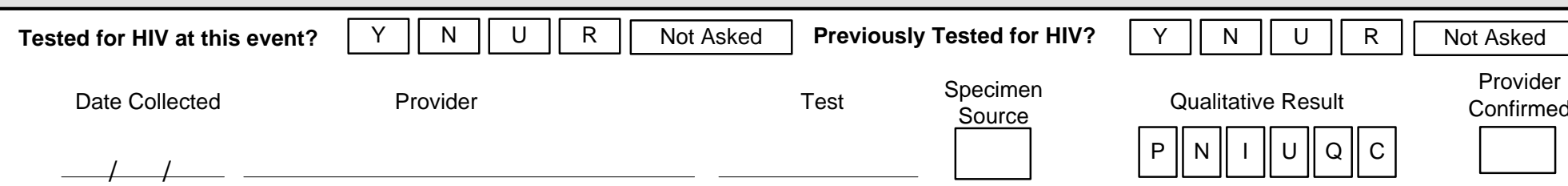

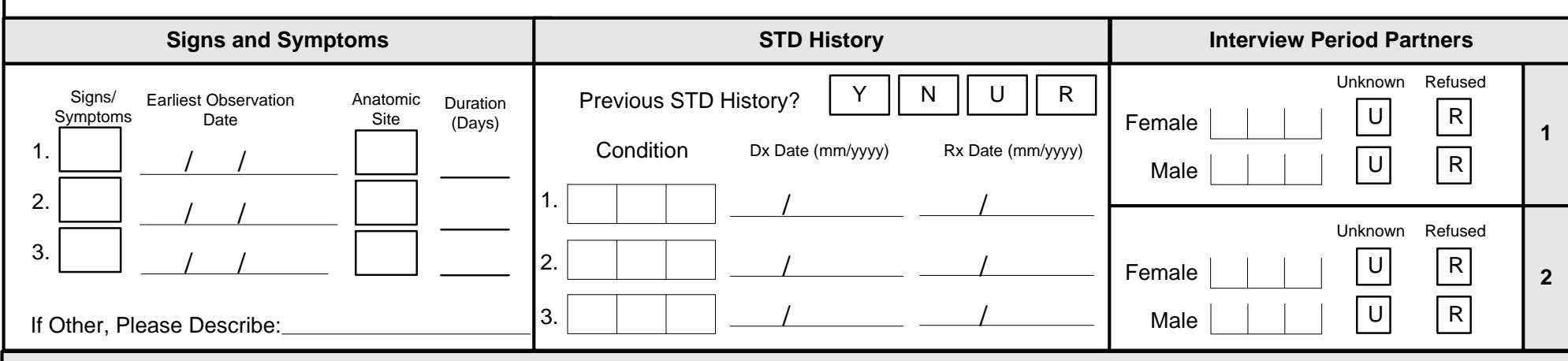

\section{Partner/Cluster Information}

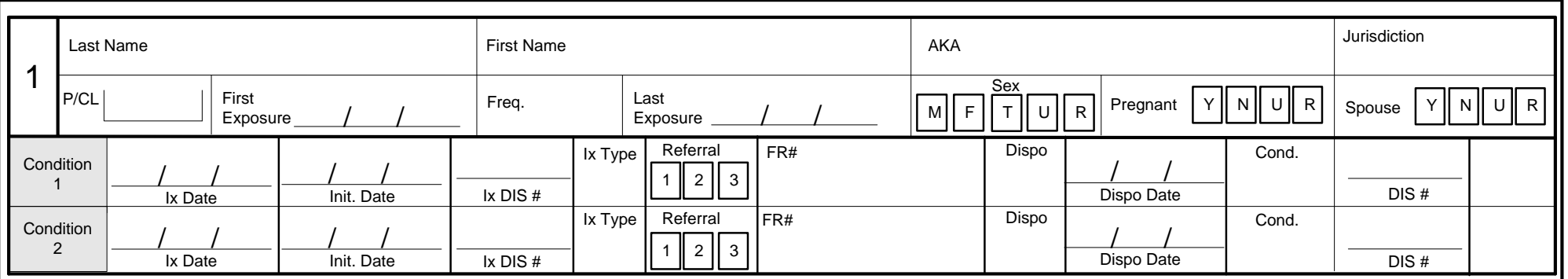

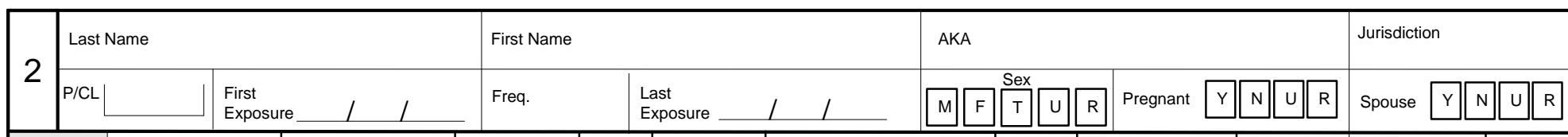

\begin{tabular}{|c|c|c|c|c|c|c|c|c|c|c|c|}
\hline \multirow{2}{*}{$\begin{array}{c}\text { Condition } \\
1 \\
\end{array}$} & & & & \multirow[t]{2}{*}{ Ix Type } & \multicolumn{2}{|c|}{ Referral } & \multirow[t]{2}{*}{ FR\# } & \multirow[t]{2}{*}{ Dispo } & & \multirow[t]{2}{*}{ Cond. } & \\
\hline & $1 \quad 1$ & I I I & IXDIS \# & & \begin{tabular}{l|l|}
1 & 2 \\
\end{tabular} & 3 & & & $\frac{1}{\text { Dispo Date }}$ & & DIS \# \\
\hline Condition & & & & Ix Type & Referra & & FR\# & Dispo & 1 & Cond. & \\
\hline 2 & Ix Date & Init. Date & Ix DIS \# & & \begin{tabular}{l||l|}
1 & 2 \\
\end{tabular} & 3 & & & Dispo Date & & DIS \# \\
\hline
\end{tabular}

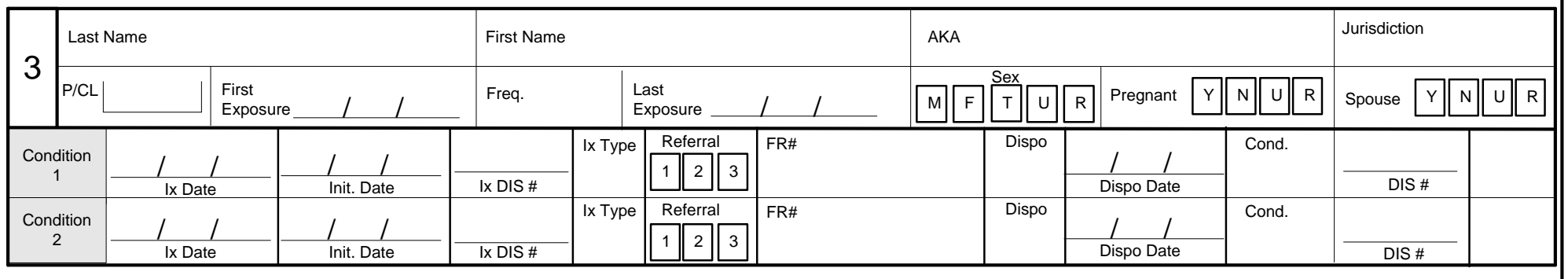

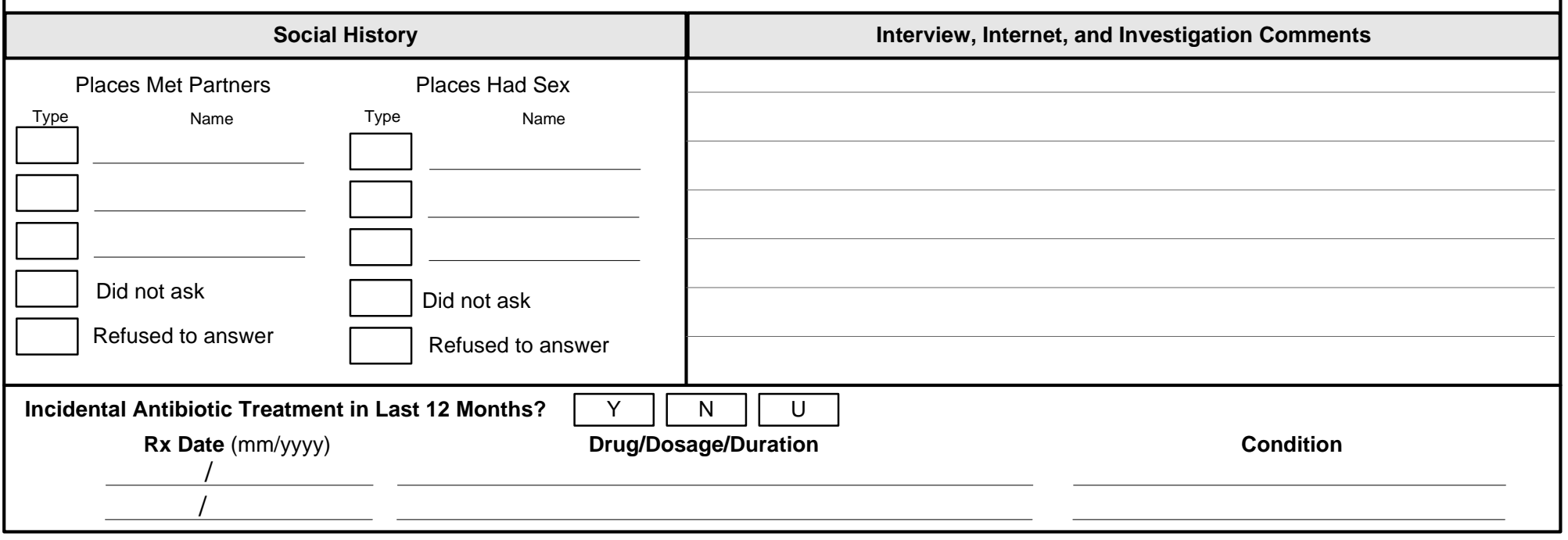




\section{Interview Record for Gonorrhea/Chlamydia}

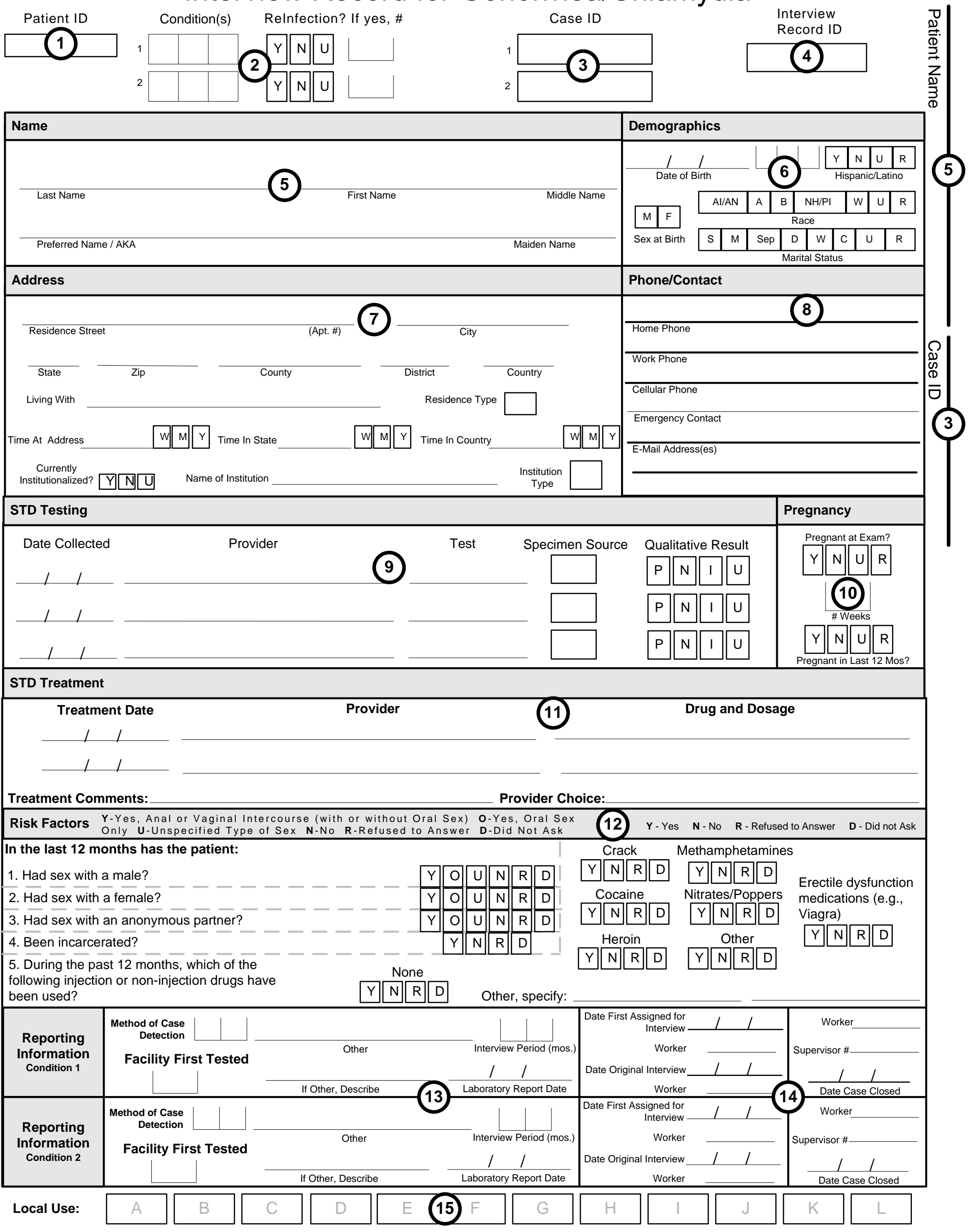




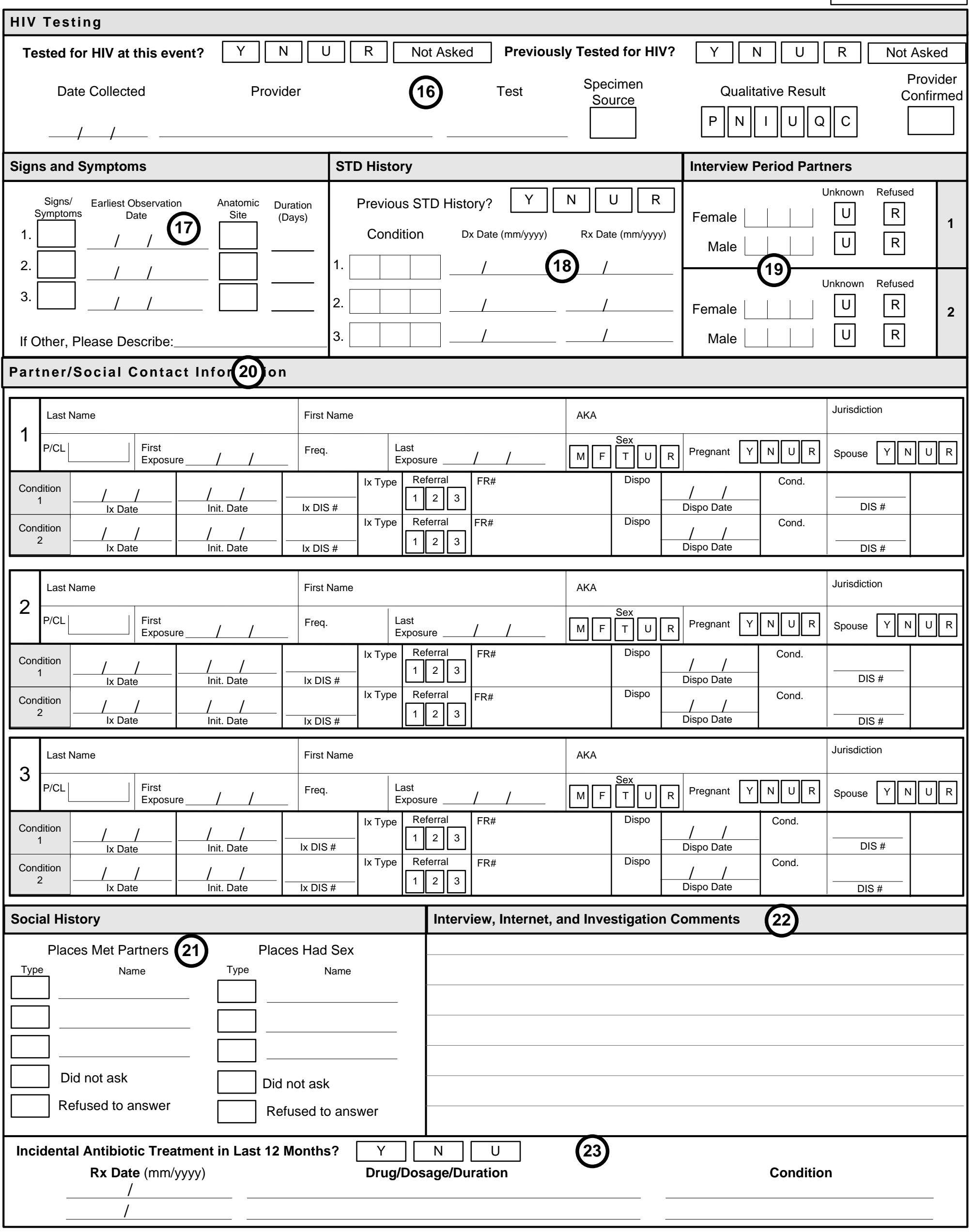


Interview Record Instructions (GC/CT Version)

The Centers for Disease Control and Prevention (CDC) Interview Record is primarily designed for use by state and local Disease Intervention Specialists (DIS) who interview individuals with sexually transmitted diseases (STDS), including HIVIAIDS and other related conditions, and conduct sex and needle sharing partner notifications and referrals. This instrument is meant to assist DIS and their managers in documenting and evaluating case management activities according to local program priorities and/or regulations. State and local program priorities and regulations will also determine what types of information should be documented and retained on this form. A small portion of the information recorded within this document will be transmitted to CDC.

Though some data elements (excluding personal identifiers, i.e. names, addresses, telephone numbers, etc.) will be forwarded to CDC, this form does not represent a document that is to be used for federal data collection purposes in its entirety. Also, this form and accompanying documentation is an example of an Interview Record that can be used by local and state programs and is made available for local and state program use and adaptation.

These instructions describe how to complete the interview record form. Each numbered item in the instructions corresponds to a number on the sample interview record form.

NOTE: The "Month/Day/Year" (MM/DD/YYYY) format should be utilized for all date fields on this record, unless otherwise specified.

(1) Patient ID Number: Document the patient ID for this person, if known or applicable.

NOTE: If using a computerized system, this number may be assigned by the software system.

Condition: Document the specific disease code for the diagnosed and/or interviewed condition. Conditions will be interviewed for and/or documented on an interview form depending on local programmatic procedures and policies in place. If one of the conditions being interviewed is syphilis or HIV related, this is not the proper interview record to be completed.

030 - HepB acute w/o delta
031 - HepB acute w/ delta
033 - HepB chronic w/o delta
034 - HepB chronic w/ delta
042 - Hepatitis delta
051 - Hepatitis C, acute
053 - Hepatitis E
054 - Hepatitis C, chronic
070 - Hepatitis, unknown

100 - Chancroid
200 - Chlamydia
300 - Gonorrhea (uncomplicated)
350 - Resistant Gonorrhea
400 - Non-Gonoccocal Urethritis (NGU)
450 - Mucopurulent Cervicitis (MPC)
490 - PID Syndrome
500 - Granuloma Inguinale
600 - Lymphogranuloma Venereum (LGV)

Second Condition: Document a $2^{\text {nd }}$ Condition, if one exists, using same list as above for disease(s) interviewed.

Reinfection? If yes, \#: Document if the interviewed condition is a Relnfection of same condition within the last 12 months. If yes, document the number of times, including current infection, patient has been infected with this conditition in the last 12 months.

(3)

Case ID(s) Number: Document the case ID number(s) for the corresponding condition(s).

NOTE: If using a computerized system, this number may be assigned by the software system.

(4)

Interview Record ID: Document the interview record number for this case, if known. This number is used for data processing/control purposes to link related cases.

NOTE: If using a computerized system, this number may be assigned by the software system.

\section{Name}

Name: Document the patient's last, first and middle names, any aliases or nicknames (AKAs), and maiden name (as applicable). 


\section{Demographics}

Date of Birth: Document the patient's date of birth. Leave blank if unknown.

Age: Document the patient's age at the time of initial exam for the earliest condition reported on this interview record. Document ' 0 ' if age is less than one year or '999' if unknown.

Hispanic or Latino*: Place an "X" in the appropriate box to identify the ethnic group with which the patient self identifies. Hispanic origin means a person of Spanish, Cuban, Mexican, Puerto Rican, South or Central American, or other Spanish culture or origin, regardless of race.

$$
\begin{aligned}
& \text { Y - Yes, Hispanic/Latino } \\
& \text { N - No, not Hispanic/Latino } \\
& \text { U - Unknown } \\
& \text { R - Refused to answer }
\end{aligned}
$$

Sex at Birth: Place an "X" in the appropriate box for the patient's biologic sex at birth: male or female. Leave blank if unknown.

Race*: Place an "X" in as many boxes as applicable. Base on the racial group(s) with which the patient self identifies. Al/AN (American Indian or Alaska Native): A person having origins in any of the original peoples of North and South America (including Central America).

A (Asian): A person having origins in any of the original peoples of the Far East, Southeast Asia, or the Indian subcontinent including, for example, Cambodia, China, India, Japan, Korea, Malaysia, Pakistan, the Philippine Islands, Thailand, and Vietnam.

B (Black or African American): A person having origins in any of the black racial groups of Africa. NH/PI (Native Hawaiian or Other Pacific Islander): A person having origins in any of the original peoples of Hawaii, Guam, Samoa, or other Pacific Islands.

W (White): A person having origins in any of the original peoples of Europe, the Middle East, or North Africa.

$\mathbf{U}$ (Unknown): The patient could not answer this question for any reason.

$\mathbf{R}$ (Refused): The patient refused to answer this question.

Marital Status: Place an " $X$ " in the appropriate box indicating marital status at the time of the interview or morbidity report.
S - Single, Never Married
W - Widowed
M - Married
C - Cohabitation
SEP - Separated
U - Unknown
D - Divorced
$\mathbf{R}$ - Refused to Answer

\section{Address}

Address: Document the complete address where the patient currently resides. If the patient is currently institutionalized (e.g., in jail, in a group home, in a mental health facility, etc.), do not document the address of the institution unless it is determined that the condition was acquired in the institution. Include apartment number, city, county, 2-letter abbreviation for the state, 5-digit zip code, district or region (if applicable), and country for the address where the patient resides. Note: Work address(es) can be documented within the

NOTE: If this is a temporary address, record the patient's permanent address and any other interview period addresses in the Comments section. For an institutionalized person, list the last known address where the person resided.

NOTE: Work address(es) can be documented within the comment section.

Living With: Document the RELATIONSHIP (such as spouse, parents, sibling, roommate, etc., not the name) of those living with the patient.

Residence Type: Document the appropriate code in the box for the type of residence for the above address.
A - Apartment
$\mathrm{N}$ - Homeless
B - Mobile Home
$\mathrm{O}$ - Other 


\section{Address}

$$
\begin{aligned}
& \text { C - Migrant Camp } \\
& \text { D - Dorm } \\
& \text { G - Group Home } \\
& \text { H - House/Condo } \\
& \text { J - Jail } \\
& \text { M - Hotel/Motel }
\end{aligned}
$$

Time At Address: Document the length of time the patient has lived at the current address, in this state/territory, and in the country. Also, place an " $X$ " in the appropriate box to indicate whether the time at the corresponding location is in (W) weeks, (M) months, or ( $\mathbf{Y})$ years. If length of time is unknown, please document "UNK".

Currently Institutionalized?: Place an " $X$ " in the appropriate box to indicate if the patient is institutionalized (i.e., in jail, in a group home, in a mental health facility, etc.). If institutionalized, document the name of the facility.

Institution Type: Document the appropriate code in the box for the type of facility where the patient is currently institutionalized.

$$
\begin{aligned}
& \text { G - Group Home } \\
& \text { J - Jail } \\
& \text { O - Other } \\
& \text { P - Prison }
\end{aligned}
$$
Q - Mental Health Center
$\mathrm{R}$ - Rehabilitation Center
$X$ - Drug Treatment/Detox Center
$\mathrm{Y}$ - Juvenile Detention

\section{Phone/Contact}

8 Phone/Contact: Document the phone number(s) where the patient can be reached and the patient's e-mail address(es) if applicable. Include an emergency contact name, phone number, and relationship to patient, if available.

\section{STD Testing}

9 Test Results: Summarize all STD lab results relevant to this case, noting at least the last negative result, the first positive result, and the most recent test if applicable.

NOTE: HIV testing is not to be documented here but in HIV Testing Section.

NOTE: Hepatitis testing can be documented within this section.

Date Collected - Document the date the specimen was obtained from the patient.

Provider - Document the specific name or code of the provider (physician, clinic, hospital, etc.) who ordered the testing.

Test - Document the name (or type) of the test performed (e.g., RPR, TP-PA, darkfield, etc.). Ensure that the test type and condition (disease) being asked for is clear.

Source - Document the code from the list below for the source of specimen collection.
01 - Cervix/Endocervix
02 - Lesion-Genital
03 - Lesion-Extra Genital
04 - Lymph Node Aspirate
05 - Oropharynx
06 - Ophthalmia/Conjunctiva
07 - Other
08 - Other Aspirate

09 - Rectum

10 - Urethra

11 - Urine

12 - Vagina

13 - Blood/Serum

14 - Cerebrospinal fluid (CSF)

88 - Not Applicable

99 - Unknown

Qualitative Results - Place an " $X$ " in the appropriate box to indicate the test result(s).

$$
\begin{aligned}
& \text { P - Positive } \\
& \text { N - Negative }
\end{aligned}
$$




\section{Pregnancy}

Pregnant at Exam?*: Place an " $X$ " in the appropriate box to indicate the patient's pregnancy status at initial exam for the condition(s) documented on this interview record. If the patient was pregnant at the time of the initial exam, document the duration of the pregnancy in weeks at exam. Y - Yes, N - No, U - Unknown, R - Refused to Answer.

Pregnant in Last 12 Months?: Determine if the patient has been pregnant during the last 12 months and place an " $X$ " in the appropriate box. If currently pregnant, a "Yes" answer indicates that the patient had another pregnancy within the past 12 months, not including her current pregnancy. Y - Yes, N - No, U - Unknown, R - Refused to Answer.

\section{STD Treatment}

(11) Treatment: Document all relevant treatment regimen(s). For the recommendations of adequate treatment, see the current CDC Treatment Guidelines.

Treatment Date - Document the date treatment was first started.

Provider - Document the name or code of the provider (physician, clinic, hospital, etc.) that provided the treatment.

Drug and Dosage - Document the name of the drug given, as well as the dosage and duration (e.g., 2.4 Bicillin $\times 3$ weeks or Doxycycline 100mg bid x 28 days).

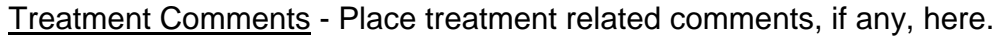

Provider Choice - Document patient's preferred provider for treatment.

\section{Risk Factors}

NOTE: Each risk factor should be addressed for the last 12 months prior to the date of the original interview. Also, for the purposes of risk assessment, sex is defined as having engaged in oral, anal and/or vaginal contact with another individual.

NOTE: For each risk $1-3$, the patient should be asked what type of sexual exposure occurred. Document the appropriate response, one response per risk factor.

$$
\begin{aligned}
& \text { Y - Yes, Anal or Vaginal Intercourse (with or without Oral Sex) } \\
& \mathbf{O}-\text { Yes, Oral Sex Only } \\
& \mathbf{U}-\text { Unspecified Type of Intercourse } \\
& \mathbf{N}-\text { No Sexual Exposure } \\
& \mathbf{R}-\text { Refused to Answer } \\
& \text { D - Did Not Ask }
\end{aligned}
$$

Anonymous - a sex partner whose name is unknown (e.g., met at a sex party, website, bathhouse, etc.).

Drug Use Behaviors: Document the appropriate response of $Y$ - Yes, N - No, R - Refused to Answer, or D - Did Not Ask. Please place an "X" in the appropriate box(es) for all recreational drug types used within the last 12 months.

\section{Condition(s) Reporting Information}

Method of Case Detection: Document the specific method of case detection code, i.e., how the patient first came to the attention of the health department, for each condition(s).

20 - Screening: An asymptomatic patient was identified through screening (routine testing of populations who are asymptomatic in order to identify those with disease). Examples of screening programs include health department outreach to high-risk populations (e.g., commercial sex-workers), HIV care clinics, family planning, blood donation, corrections-based, and prenatal. This includes STD and other health department 
clinic visits by a client who tests positive for a condition with which they were unaware (e.g., asymptomatic walk-ins) of before being seen at the clinic.

21 - Self-Referred: Refers to patient who sought health services because of signs of an STD and were subsequently tested for the disease being reported. This includes symptomatic STD clinic testing.

22 - Patient Referred Partner: Patient referred by another infected person. This may be a named or unnamed partner. No health department involvement was necessary for this referral.

23 - Health Department Referred Partner: This patient is a named partner of a known case. Patient identified through DIS, or other health department personnel, activity following an interview of another known case. The health department was involved in the referral of this individual (e.g., the DIS contacted, called, visited, sent letter, etc., the patient to inform them of their need to be tested).

24 - Cluster Related: Patient was originally identified as a Social Contact (Suspect) or Associate. Cluster brought to the attention of the program as a result of a DIS interview.

88 - Other: In the event of NONE of the above being applicable, accurately describe how the patient came to the attention of the health department.

Interview Period: Document the interview period in months for each condition.

Type Facility First Tested: Document the specific type of facility code where the patient was first tested for each condition. If ' 88 ' (Other) please describe as specifically as possible in the space provided.
01 - HIV Counseling/Testing Site
02 - STD Clinic
03 - Drug Treatment
04 - Family Planning
05 - RETIRED
06 - TB Clinic
07 - Other HD Clinic
08 - Private MD/HMO
09 - RETIRED
10 - Hospital (ER)
11 - Correctional facility
12 - Lab

13 - Blood Bank

14 - Labor and Delivery

15 - Prenatal

16 - Job Corps

17 - School-based Clinic

18 - Mental Health Services

29 - Hospital (Other)

66 - Indian Health Services

77 - Military

88 - Other

99 - Unknown

Date of Laboratory Report: Document the date the first laboratory report related to the interviewed condition documented on this interview record was initially received at the health department (or any authorized public health agency, e.g., the STD clinic) for each condition(s).

\section{Case Information}

Date First Assigned for Interview: Document the date this case was initially assigned for interview and the worker number of the DIS to whom it was assigned for each condition.

Date Original Interview: Document the date of the initial interview and the worker number of the DIS that performed the interview for each condition.

Date Case Closed: Document the date of case closure as well as the worker numbers of the investigating DIS and supervisor, if applicable, responsible for the management of this case for each condition(s). The determination of closure should be made by the DIS and supervisor, if applicable, after all reasonable efforts have been expended on the case.

Local Use: This area is provided for special data collection needs of individual program areas.

\section{HIV Testing}

Tested for HIV at this event?: Place an " $X$ " in the appropriate box to indicate whether the patient was tested for HIV at the time of the initial screening that led to this reported condition(s). Y - Yes, N - No, U - Unknown, R - Refused to Answer, or Not Asked. 
NOTE: Relevant HIV testing and interview may occur on the same day. Also, the answer "No" for Tested for HIV at this event includes opt-out/routine HIV testing in settings where HIV pretest counseling is not conducted.

Previously Tested for HIV?: Place an " $X$ " in the appropriate box to indicate whether the patient has tested for HIV prior to the event that led to the Original Interview. $\mathrm{Y}-\mathrm{Yes}, \mathrm{N}-\mathrm{No}, \mathrm{U}-$ Unknown, R - Refused to Answer, or Not Asked.

HIV Test Results: Place the most recent or relevant HIV lab results. Document the date collected, the provider name or code who ordered the test, the name of the test, the source, and the qualitative result (see item \#9 for codes).

Provider Confirmed - Place a 'Y' for 'Yes' if HIV test result(s) has been provider confirmed by record search or direct contact with a provider. Place an 'N' for 'No' if based on interviewee responses only.

\section{Signs and Symptoms}

Signs and Symptoms: Determine if there are signs or symptoms related to the condition(s) documented on this interview record. This includes all symptoms experienced by the patient and signs observed by a clinician. Additional signs and symptoms can be documented within the Interview/Investigation Comments.

Signs/Symptoms - Document the code for each sign/symptom observed on exam or described:
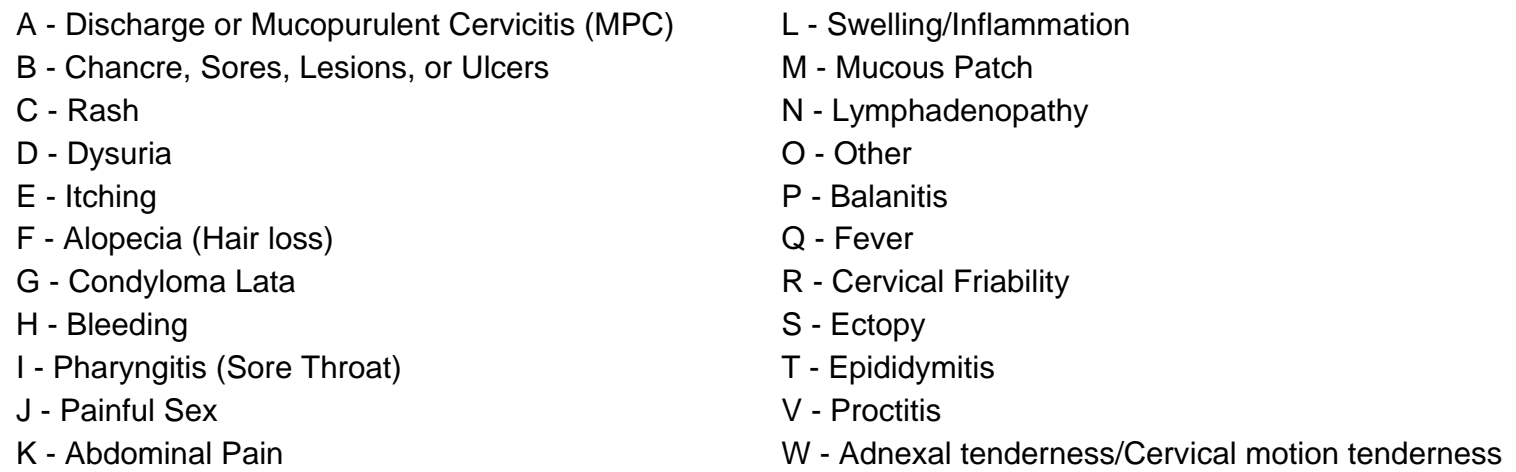

Earliest Observation Date - Document the earliest date the symptom was first experienced by the patient and/or the date the sign was first observed by a clinician.

Anatomic Site* - Document the code indicating the anatomic site of the sign/symptom.

$\begin{array}{ll}\text { A - Anus/Rectum } & \text { H - Eye-Conjunctiva } \\ \text { B - Penis } & \text { I - Head } \\ \text { C - Scrotum } & \text { J - Torso } \\ \text { D - Vagina } & \text { K - Extremities (Arms, Legs, Feet, Hands) } \\ \text { E - Cervix } & \text { N - Not Applicable } \\ \text { F - Naso-Pharynx } & \text { O - Other } \\ \text { G - Mouth/Oral Cavity } & \text { U - Unknown }\end{array}$

Duration (Days) - Document the number of days signs/symptoms were present. Document "99" if unknown.

If Other, Please Describe - if sign/symptom code "O" is used, please describe in the space provided.

\section{STD History}

STD History*: Place an " $X$ " in the appropriate box indicating if the patient has a history of STDs (prior to the condition(s) documented on this interview record). HIV testing history should be documented in the HIV Testing section.

Y - Yes, patient has a history of STD

$\mathbf{N}$ - No, patient has never had a prior STD

$\mathbf{U}$ - Unknown if patient has had a prior STD

$\mathbf{R}$ - Patient refused to answer any questions regarding prior STD History

If 'Yes', document the condition code(s), diagnosis date(s) (MM/YYYY), and treatment date(s) (MM/YYYY) in the space provided. 
030 - HepB acute w/o delta

031 - HepB acute w/ delta

033 - HepB chronic w/o delta

034 - HepB chronic w/ delta

042 - Hepatitis delta

051 - Hepatitis C, acute

053 - Hepatitis E

054 - Hepatitis C, chronic

070 - Hepatitis, unknown
100 - Chancroid
200 - Chlamydia
300 - Gonorrhea (uncomplicated)
350 - Resistant Gonorrhea
400 - Non-Gonoccocal Urethritis (NGU)
450 - Mucopurulent Cervicitis (MPC)
490 - PID Syndrome
500 - Granuloma Inguinale
600 - Lymphogranuloma Venereum (LGV)

\section{Interview Period Partners}

Interview Period Partners: Document the total number of female, male, and transgender sex partners claimed by this patient during the interview period for each Condition(s). Note that this includes initiated partners, marginal partners, and anonymous partners. For example, the patient may claim 10 sex partners during the 3-month interview period (Primary Syphilis), while there is only enough information to initiate 3; the total of 10 (rather than 3) should be documented for interview period partners. Select 'unknown' if patient is unsure or 'refused' if the patient would not answer the question. Document " 0 " if there are no partners for corresponding gender field.

\section{Partner/Cluster Information}

This section of the form is used to record all interview activity and the results of investigations regarding partners and clusters. Guidelines for completing the partner/cluster initiation section are:

NOTE: If a patient is interviewed, complete at least one partner/cluster section. If no partners/clusters are added, document the date of interview, the number of the DIS/worker who conducted the interview, and the type of interview conducted.

NOTE: Document only the names of sex partners, social contacts and associates for whom sufficient information has been obtained to initiate a Field Record. Information on marginal contacts and clusters should be documented in the space provided and/or on a buff.

NOTE: All re-interview or cluster activity must be listed in separate sections. Use of Re-Interview and Cluster Interview Forms are encouraged for complete documentation.

NOTE: Clusters must be named by the index patient, named contacts or named clusters to be documented on the interview record. General field screening not specifically associated with this interviewed patient should not be included in this section. Other mechanisms must be used to collect this type of screening information.

Name: Document the Last and First name and, if applicable, known aliases of the partner/cluster.

Jurisdiction: Document the county, state or country code or name for where the partner/cluster resides. Use of code or name depends on local programmatic discretion.

PICl (Partner/Cluster): Document the appropriate identifier for the specific type of partner or cluster (Suspect and/or Associate).

PARTNER - Persons having sexual activities (of any type) or sharing needles with the Index patient.

P1 - Sex Partner

P2 - Needle sharing Partner

P3 - Both Sex and Needle sharing Partner

SOCIAL CONTACT (SUSPECT) - Persons named by an infected person (e.g., the Index patient or an infected partner or cluster).

S1 - Person who has or had symptoms suggestive of the Condition(s) documented.

S2 - Person who is named as a sex partner of a known infected person.

S3 - Any other person who would benefit from an exam (i.e., someone who has engaged in a behavior that 


\section{Partner/Cluster Information}

might put them at risk).

ASSOCIATE - Persons named by an uninfected partner or cluster.

A1 - Person who has or had symptoms suggestive of the Condition(s) documented.

A2 -Person who is named as a sex partner of a known infected person.

A3 - Any other person who would benefit from an exam (i.e., someone who has engaged in a behavior that might put them at risk).

Exposure to Original Patient: Document the Index Patient's contact with the partner.

First Exposure - Document the date of the first sexual/needle-sharing exposure to the Index patient.

Freq. (Frequency) - Document the frequency (number) of sexual/needle-sharing exposure to the Index patient between the first and last (most recent) exposure. This should be described as specifically as possible: $1 \mathrm{x}=$ one time, $2 \mathrm{x} / \mathrm{wk}=$ two times a week, etc. If the frequency is unknown, document " 99 ".

Last Exposure - Document the date of the last (most recent) sexual/needle-sharing exposure.

NOTE: Exposure information should only be documented for partners of the Index patient; only what the Index patient claimed as exposure should be documented, NOT what the partners claimed as exposure.

Sex: Place an " $X$ " in the appropriate box to indicate the gender of the partner or cluster, as identified by the person being interviewed: $\mathbf{M}$ - Male, F - Female, $\mathbf{T}$ - Transgender, $\mathbf{U}$ - Unknown, $\mathbf{R}$ - Refused.

NOTE: If transgender is marked, MTF or FTM should be documented on the corresponding Cluster Interview Record.

Pregnant: Document if this partner/cluster is pregnant: $\mathbf{Y}-\mathrm{Yes}, \mathbf{N}-\mathrm{No}, \mathbf{U}$ - Unknown, $\mathbf{R}$ - Refused to Answer.

Spouse: Document if this partner/cluster is the Index patient's spouse: $\mathbf{Y}-$ Yes, $\mathbf{N}-\mathbf{N o}, \mathbf{U}$ - Unknown, $\mathbf{R}$ - Refused to Answer.

IX Date (Interview Date): Document the date the original interview, re-interview or cluster interview was performed. Document interview dates where no partners or clusters are initiated as well using.

Init. Date (Initiation Date): Document the date this partner/cluster was initiated for field investigation.

IX DIS \# (Interview DIS): Document the worker number of the DIS who conducted the interview for each condition (if multiple conditions). Also, document the worker number if no contacts or clusters are initiated.

Ix Type (Interview Type): Document the type of interview that was the source of the partner/cluster information.

O - Original Interview - the initial interview with an infected patient.

$\mathbf{R}$ - Re-Interview - any interview after the Original Interview of an infected patient.

C - Cluster Interview - any interview of a partner or cluster regarding the index case.

$\mathbf{U}$ - Unable to interview - (may include situations where the Index patient was not interviewed, but sex partners and/or clusters were initiated from other activities).

Referral: This describes how initiated partners and clusters are brought to examination and/or treatment. This documentation will take place at the time of the disposition (closure) of the field record. Document the type of referral for each condition.

1 - Provider: DIS or other health department staff were involved in the referral of this partner/cluster.

2 - Patient (Client): No health department involvement in the referral of this partner/cluster.

3 - Dual (Contract): A combination of provider and patient effort to bring contact/cluster to services.

FR \# (Field Record Number): Document the entire field record number(s) for the partner/cluster initiated. This number is located in the lower left corner of the CDC form 73.2936S, or may be generated by the software system. 


\section{Partner/Cluster Information}

Dispo (Disposition): Document the STD or HIV disposition code from the field record for each Condition(s):

\section{STD Dispositions}

A - Preventative Treatment

B - Refused Preventative Treatment

C - Infected, Brought to Treatment

D - Infected, Not Treated

E - Previously Treated for This Infection

F - Not Infected

G - Insufficient Information to Begin Investigation

H - Unable to Locate

J - Located, Refused Examination and/or Treatment

K - Out Of Jurisdiction

L - Other
HIV Dispositions

1 - Previous Positive

2 - Previous Negative, New Positive

3 - Previous Negative, Still Negative

4 - Previous Negative, Not Re-tested

5 - Not Previously Tested, New Positive

6 - Not Previously Tested, New Negative

7 - Not Previously Tested, Not Tested Now

G - Insufficient Information to Begin Investigation

H - Unable to Locate

J - Located, Refused Counseling and/or Testing

$\mathrm{K}$ - Out Of Jurisdiction

L - Other

Dispo Date (Disposition Date): Document the appropriate date as it relates to the following examination or treatment situation for each Condition(s).

Newly Examined and Treated - Use the date of treatment.

Newly Examined, not Treated - Use the date of examination.

Previously Examined and/or Treated - Use the date the partner/cluster investigation is closed (i.e., the date the investigator became aware of the previous examination and/or treatment).

Not Examined - Use the date the investigation is closed.

NOTE: A partner/cluster CAN NOT be dispositioned before it is initiated. Therefore, if examination and/or treatment occurred prior to the partner/cluster being initiated (e.g., disposition ' $E$ ' or ' $A$ '), the disposition date can be no earlier than the initiation date.

Cond. (Condition): If partner/cluster is dispositioned as infected, whether previously or currently, document the diagnosis code for the condition.

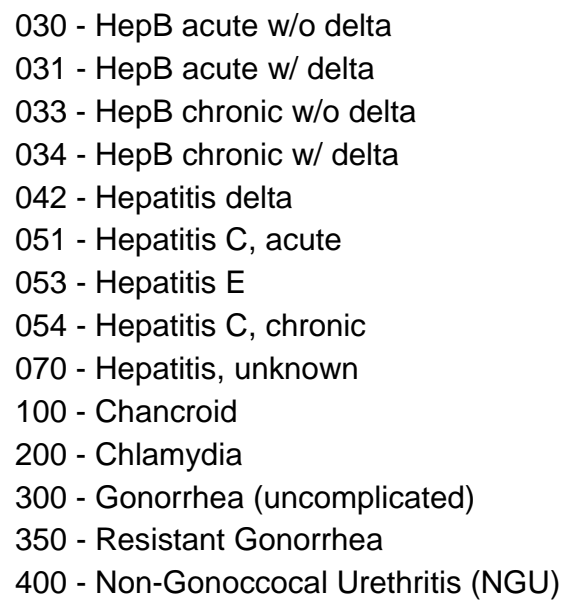

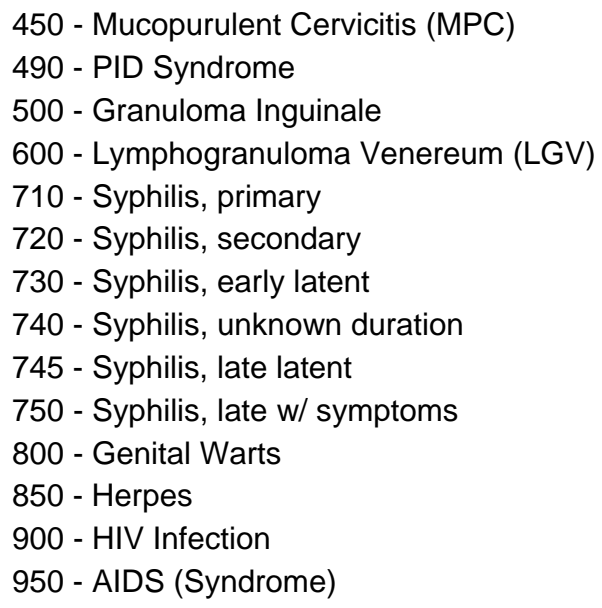

DIS \#: Document the worker number of the DIS who brought this partner or cluster to disposition for each Condition(s).

\section{Social History}

Places Met Partners: Document the codes for the types of places where the patient met sex partners within the last 12 months (document as many as apply):

\begin{tabular}{|l|l|l|}
\hline A - Adult Book Store/Cinema & J - Jail/Prison & S - Partner's Home \\
B - Bars & K - Clubs & T - Street \\
C - Cruising in Automobile & L - Beach & U - Circuit Party \\
\hline
\end{tabular}




\begin{tabular}{|l|l|l|}
\hline D - Dance Halls & M - Motel/Hotel & V - Cruise (Boat) \\
E - Escort Services & N - Shopping Mall & W - Work \\
F - Baths/Spas/Resorts & O - Other & X - Park/Rest Area \\
G - Place of Worship & P - Project/Shelter & \\
H - Home & Q - School & \\
I - Chat Rooms/Lines/Email/Internet & R - Gyms/Health Clubs & \\
\hline
\end{tabular}

Document the names (or descriptions) of places the patient goes to meet sex partners. Document 'did not ask' or 'refused to answer' if applicable. If additional space is needed, document within the Interview/Investigation Comments (item 23).

Places had Sex: Document the codes (from above list) for the types of places where the patient had sex with partners within the last 12 months (document as many as apply); document the names (or descriptions) of the places the patient had sex with partners. Document 'did not ask' or 'refused to answer' if applicable. If additional space is needed, document within the Interview/Investigation Comments.

\section{Interview, Internet, and Investigation Comments}

Interview/Investigation Comments: This section is provided to record, in a narrative fashion, any additional information not included in the interview record, any relevant information discovered in the course of the investigation (such as attitude of the patient, if he or she was high/intoxicated, etc.), or to note any inconsistencies during the interview or DIS analysis of interview information. Also use this space to document any related internet use information, including alternate email addresses, instant messenger usernames, chat sites, etc. Also, note whether there were STD Clinic or provider accessability or availability issues that affected service to the patient.

\section{Incidental Antibiotic Treatment in Last 12 Months}

Place an "X" in the appropriate box, $Y$ - Yes, N - No, $U$ - Unknown. If incidental antibiotic treatment occurred (that being an antibiotic that the patient did not receive to specifically treat this condition), document the date (MM/YYYY) the treatment began and the drug, dosage and duration used, and for what condition the treatment was prescribed, if known. If the date the treatment began is unknown, document "99/9999". 